\title{
Light hadrons from lattice QCD with light $(u, d)$, strange and charm dynamical quarks
}

\section{ETM collaboration}

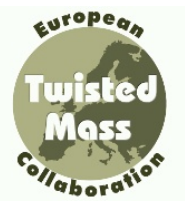

R. Baron, ${ }^{a}$ Ph. Boucaud, ${ }^{b}$ J. Carbonell, ${ }^{c}$ A. Deuzeman, ${ }^{d}$ V. Drach, ${ }^{c}$ F. Farchioni, ${ }^{e}$

V. Gimenez, ${ }^{f}$ G. Herdoiza, ${ }^{g}$ K. Jansen, ${ }^{g}$ C. McNeile, ${ }^{h}$ C. Michael, ${ }^{i}$ I. Montvay, ${ }^{j}$

D. Palao, ${ }^{k}$ E. Pallante, ${ }^{d, 1}$ O. Pène, ${ }^{b}$ S. Reker, ${ }^{d}$ C. Urbach, ${ }^{l}$ M. Wagner ${ }^{m}$ and

U. Wenger ${ }^{n}$

${ }^{a}$ CEA, Centre de Saclay, IRFU/Service de Physique Nucléaire, F-91191 Gif-sur-Yvette, France

${ }^{b}$ Laboratoire de Physique Théorique (Bât. 210), CNRS et Université Paris-Sud 11, Centre d'Orsay, 91405 Orsay-Cedex, France

${ }^{c}$ Laboratoire de Physique Subatomique et Cosmologie, 53 avenue des Martyrs, 38026 Grenoble, France

${ }^{d}$ Centre for Theoretical Physics, University of Groningen, Nijenborgh 4, 9747 AG Groningen, the Netherlands

${ }^{e}$ Institut für Theoretische Physik, Universität Münster, Wilhelm-Klemm-Straße 9, D-48149 Münster, Germany

${ }^{f}$ Dep. de Física Teòrica and IFIC, Universitat de València-CSIC, Dr.Moliner 50, E-46100 Burjassot, Spain

${ }^{g}$ NIC, DESY, Platanenallee 6, D-15738 Zeuthen, Germany

${ }^{h}$ Department of Physics and Astronomy, The Kelvin Building, University of Glasgow, G12 8QQ Glasgow, U.K.

${ }^{i}$ Division of Theoretical Physics, University of Liverpool, L69 3BX Liverpool, U.K.

${ }^{j}$ DESY, Notkestr. 85, D-22603 Hamburg, Germany

"INFN, Sez. di Roma "Tor Vergata", Via della Ricerca Scientifica 1, I-00133 Rome, Italy

${ }^{l}$ Helmholtz-Institut für Strahlen- und Kernphysik (Theorie) and Bethe Center for Theoretical Physics, Universität Bonn, 53115 Bonn, Germany

\footnotetext{
${ }^{1}$ Corresponding author.
} 
${ }^{m}$ Institut für Physik, Humboldt-Universität zu Berlin, Newtonstraße 15, D-12489 Berlin, Germany

${ }^{n}$ Albert Einstein Center for Fundamental Physics, Institute for Theoretical Physics, University of Bern, Sidlerstr. 5, CH-3012 Bern, Switzerland

E-mail: e.pallante@rug.nl

ABSTRACT: We present results of lattice QCD simulations with mass-degenerate up and down and mass-split strange and charm $\left(N_{\mathrm{f}}=2+1+1\right)$ dynamical quarks using Wilson twisted mass fermions at maximal twist. The tuning of the strange and charm quark masses is performed at two values of the lattice spacing $a \approx 0.078 \mathrm{fm}$ and $a \approx 0.086 \mathrm{fm}$ with lattice sizes ranging from $L \approx 1.9 \mathrm{fm}$ to $L \approx 2.8 \mathrm{fm}$. We measure with high statistical precision the light pseudoscalar mass $m_{\mathrm{PS}}$ and decay constant $f_{\mathrm{PS}}$ in a range $270 \lesssim m_{\mathrm{PS}} \lesssim$ $510 \mathrm{MeV}$ and determine the low energy parameters $f_{0}$ and $\bar{l}_{3,4}$ of $\mathrm{SU}(2)$ chiral perturbation theory. We use the two values of the lattice spacing, several lattice sizes as well as different values of the light, strange and charm quark masses to explore the systematic effects. A first study of discretisation effects in light-quark observables and a comparison to $N_{\mathrm{f}}=2$ results are performed.

KEYwords: Lattice QCD, Chiral Lagrangians

ArXiv EPrint: 1004.5284 


\section{Contents}

1 Introduction and main results 1

2 Lattice action $\quad 4$

2.1 Gauge action 4

2.2 Action for the light doublet 5

2.3 Action for the heavy doublet 5

$2.4 \mathcal{O}(a)$ improvement at maximal twist $\quad 6$

$\begin{array}{lll}3 & \text { Simulation details } & 7\end{array}$

3.1 Simulation ensembles $\quad 7$

3.2 Tuning to maximal twist 9

$\begin{array}{lll}3.3 & \text { Tuning of the strange and charm quark masses } & 10\end{array}$

3.4 Discretisation effects in light-quark observables 11

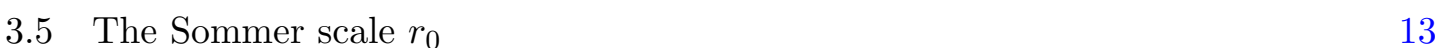

$\begin{array}{lll}3.6 & \text { Effects of isospin breaking } & 14\end{array}$

$\begin{array}{lll}3.7 & \text { Stout smeared runs } & 15\end{array}$

4 Results: $f_{\mathrm{PS}}, m_{\mathrm{PS}}$ and chiral fits 16

5 Conclusions and outlook $\quad 23$

\section{Introduction and main results}

The beginning of this century has assisted to radical improvements in theory, algorithms and supercomputer technology, leading to a far increased ability to solve non-perturbative aspects of gauge field theories in a lattice regularised framework. Following this path of improving the lattice setup, in this paper, we are reporting about our experiences and results when considering in addition to the $u, d$ light dynamical flavours also the effects of the strange and charm sea quarks. By including a dynamical charm, we are now able to directly study its contribution to physical observables and to quantify the so far uncontrolled systematic effect present in lattice QCD simulations where the charm flavour in the sea is absent.

A number of different lattice fermion formulations are being used by several lattice groups, see refs. $[1,2]$ for recent reviews. Here, we adopt a particular type of Wilson fermions, known as the Wilson twisted mass formulation of lattice QCD (tmLQCD), introduced in $[3,4]$. This approach is by now well established, with many physical results obtained with two light degenerate twisted mass flavours $\left(N_{\mathrm{f}}=2\right)$ by our European Twisted Mass (ETM) Collaboration, see refs. [5-22]. For a review see ref. [23]. In the tmLQCD 
formulation a twisted mass term is added to the standard, unimproved Wilson-Dirac operator, and the formulation becomes especially interesting when the theory is tuned to maximal twist [4]. The major advantage of the lattice theory tuned to maximal twist is the automatic $\mathcal{O}(a)$ improvement of physical observables, independently of the specific type of operator considered, implying that no additional, operator specific improvement coefficients need to be computed. Other advantages worth to mention are that the twisted mass term acts as an infrared regulator of the theory and that mixing patterns in the renormalisation procedure are expected to be simplified.

Detailed studies of the continuum-limit scaling in the quenched approximation [24-27] and with two dynamical quarks $[7,10,17,28]$ have demonstrated that, after an appropriate tuning procedure to maximal twist, lattice artefacts not only follow the expected $\mathcal{O}\left(a^{2}\right)$ scaling behaviour [4], but also that the remaining $\mathcal{O}\left(a^{2}\right)$ effects are small, in agreement with the conclusions drawn in ref. [29].

The only exception seen so far is the neutral pseudoscalar mass, which shows significant $\mathcal{O}\left(a^{2}\right)$ effects. This arises from the explicit breaking of both parity and isospin symmetry, which are however restored in the continuum limit with a rate of $\mathcal{O}\left(a^{2}\right)$ as shown in [4] and numerically confirmed in refs. [17, 30]. Moreover, a recent analysis suggests that isospin breaking effects strongly affect only a limited set of observables, namely the neutral pion mass and kinematically related quantities [31, 32].

In this paper we report on simulations with twisted mass dynamical up, down, strange and charm quarks. We realise this by adding a heavy mass-split doublet $(c, s)$ to the light degenerate mass doublet $(u, d)$, referring to this setup as $N_{\mathrm{f}}=2+1+1$ simulations. This formulation was introduced in [33,34] and first explored in [35]. As for the mass-degenerate case, the use of lattice action symmetries allows to prove the automatic $\mathcal{O}(a)$ improvement of physical observables in the non-degenerate case [33, 34]. First accounts of our work were presented at recent conferences [36, 37]. Recently, results with $N_{\mathrm{f}}=2+1+1$ staggered fermions have been reported in [38-40], while numerous studies are presently being performed with $N_{f}=2$ and $2+1$ flavours [41-48]. The inclusion of the strange and charm degrees of freedom allows for a most complete description of light hadron physics and eventually opens the way to explore effects of a dynamical charm in genuinely strong interaction processes and in weak matrix elements.

Here, we concentrate on results in the light-quark sector using the charged pseudoscalar mass $m_{\mathrm{PS}}$ and decay constant $f_{\mathrm{PS}}$ as basic observables involving up and down valence quarks only. In figure 1 we show the dependence of (a) $m_{\mathrm{PS}}^{2} / 2 B_{0} \mu_{l}$ and (b) $f_{\mathrm{PS}}$ as a function of the mass parameter $2 B_{0} \mu_{l}$, together with a fit to $\mathrm{SU}(2)$ chiral perturbation theory $(\chi \mathrm{PT})$ at the smallest value of the lattice spacing of $a \approx 0.078 \mathrm{fm}$ and lattice gauge coupling $\beta=1.95$. We summarise the fit results for the low energy constants in table 1 . These are the main results of this paper.

A comparison between data obtained with $N_{\mathrm{f}}=2+1+1$ and $N_{\mathrm{f}}=2$ flavours of quarks see sections 3.4 and 4, and ref. [17] - reveals a remarkable agreement for the results involving light-quark observables such as the pseudoscalar mass and decay constant or the nucleon mass. This provides a strong indication in favour of the good quality of our data in this new setup. In particular, barring cancellations due to lattice discretisation errors, these 


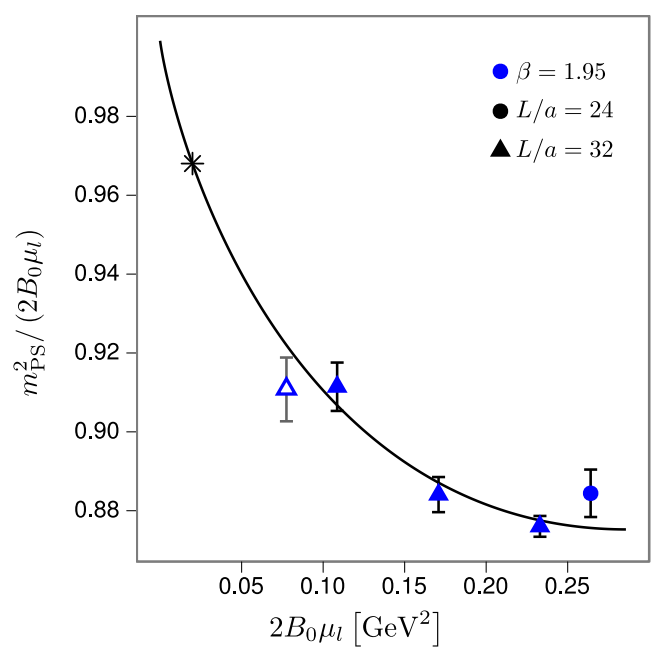

(a)

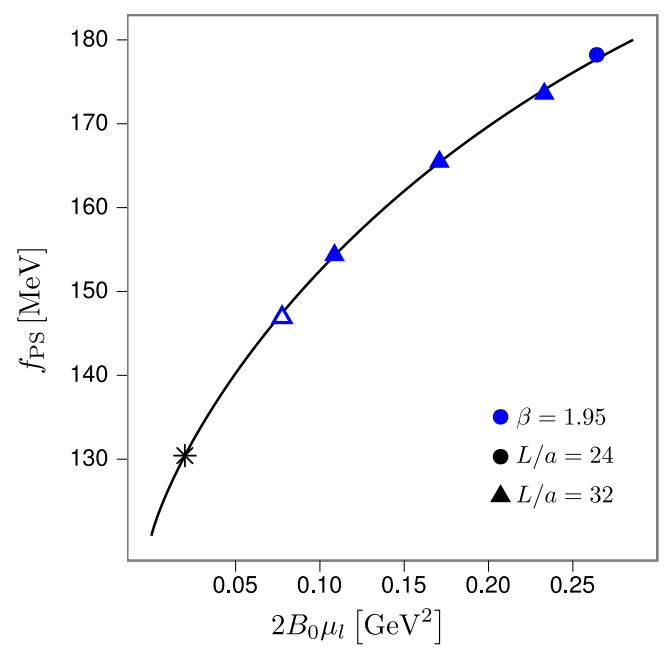

(b)

Figure 1. (a) The charged pseudoscalar mass ratio $m_{\mathrm{PS}}^{2} /\left(2 B_{0} \mu_{l}\right)$ and (b) the pseudoscalar decay constant $f_{\mathrm{PS}}$ as a function of $2 B_{0} \mu_{l}$ fitted to $\mathrm{SU}(2)$ chiral perturbation theory, see table 1 . The scale is set by the value of $2 B_{0} \mu_{l}$ at which the ratio $f_{\mathrm{PS}}^{[L=\infty]} / m_{\mathrm{PS}}^{[L=\infty]}$ assumes its physical value [49] $f_{\pi} / m_{\pi}=130.4(2) / 135.0$ (black star). The lattice gauge coupling is $\beta=1.95$ and the twisted light quark mass ranges from $a \mu_{l}=0.0025$ to 0.0085 , see eq. (2.3) for its definition, corresponding to a range of the pseudoscalar mass $270 \lesssim m_{\mathrm{PS}} \lesssim 490 \mathrm{MeV}$. The kaon and $D$ meson masses are tuned to their physical value, see table 4 . The lightest point (open symbol) has not been included in the chiral fit, see the discussion in section 3.2 .

results would suggest that the dynamical strange and charm degrees of freedom do not induce large effects in these light-quark observables. In the $N_{\mathrm{f}}=2$ case, data collected at four values of the lattice spacing have allowed us to properly quantify all systematic errors present in the determination of light-quark observables [17]. In this first work with $N_{\mathrm{f}}=2+1+1$ flavours, we consider data at two close values of the lattice spacing, while we defer to a forthcoming publication the inclusion of additional ensembles at a significantly lower lattice spacing and a more complete analysis of the systematic effects.

The rest of this paper is organised as follows. In section 2 we describe the gauge action and the twisted mass fermionic action for the light and heavy sectors of the theory. The realisation of $\mathcal{O}(a)$ improvement at maximal twist is also presented. In section 3 we define the simulation parameters, describe the tuning to maximal twist as well as the tuning of the strange and charm quark masses and the relevance of discretisation effects. Section 4 includes a discussion of the fits to $\mathrm{SU}(2) \chi \mathrm{PT}$ also for data on a slightly coarser lattice, $a \approx 0.086 \mathrm{fm}$, and provides a first account of systematic uncertainties. Our conclusions and future prospects are summarised in section 5 . 


\begin{tabular}{|lr|}
\hline & $\beta=1.95$ \\
\hline $\bar{l}_{3}$ & $3.70(7)(26)$ \\
$\bar{l}_{4}$ & $4.67(3)(10)$ \\
$f_{0}[\mathrm{MeV}]$ & $121.14(8)(19)$ \\
$f_{\pi} / f_{0}$ & $1.076(2)(2)$ \\
$2 B_{0} \mu_{u, d} / m_{\pi}^{2}$ & $1.032(21)(3)$ \\
$\left\langle r^{2}\right\rangle_{s}^{\mathrm{NLO}}\left[\mathrm{fm}^{2}\right]$ & $0.724(5)(23)$ \\
\hline$r_{0}^{\chi} / a(\beta=1.95)$ & $5.71(4)$ \\
$r_{0}^{\chi}(\beta=1.95)[\mathrm{fm}]$ & $0.447(5)$ \\
$a(\beta=1.95)[\mathrm{fm}]$ & $0.0782(6)$ \\
\hline
\end{tabular}

Table 1. Results of the fits to $\mathrm{SU}(2) \chi \mathrm{PT}$ for the ensemble at $\beta=1.95$. Predicted quantities are: the low energy constants $\bar{l}_{3,4}$, the charged pseudoscalar decay constant in the chiral limit $f_{0}$, the mass ratio $2 B_{0} \mu_{l} / m_{\mathrm{PS}}^{2}$ at the physical point and the pion scalar radius $\left\langle r^{2}\right\rangle_{s}^{\mathrm{NLO}}$. The first quoted error is from the chiral fit at $\beta=1.95$, the second error is the systematic uncertainty that conservatively accommodates the best fitted central values of the three fits reported in table 9 , section 4 . The small error on the quoted lattice spacing comes exclusively from the fit at $\beta=1.95$. The scale is set by fixing the ratio $f_{\mathrm{PS}}^{[L=\infty]} / m_{\mathrm{PS}}^{[L=\infty]}=f_{\pi} / m_{\pi}=130.4(2) / 135.0$ to its physical value [49]. The chirally extrapolated Sommer scale $r_{0}^{\chi}$ is determined separately and not included in the $\chi \mathrm{PT}$ fits. For a comparison with the $N_{\mathrm{f}}=2$ ETMC results, see [17].

\section{Lattice action}

The complete lattice action can be written as

$$
S=S_{g}+S_{l}+S_{h}
$$

where $S_{g}$ is the pure gauge action, in our case the so-called Iwasaki action [50, 51], $S_{l}$ is the twisted mass Wilson action for the light doublet $[3,4]$ and $S_{h}$ the one for the heavy doublet [33, 34].

\subsection{Gauge action}

The Iwasaki gauge action $[50,51]$ includes besides the plaquette term $U_{x, \mu, \nu}^{1 \times 1}$ also rectangular $(1 \times 2)$ Wilson loops $U_{x, \mu, \nu}^{1 \times 2}$

$$
S_{g}=\frac{\beta}{3} \sum_{x}\left(b_{0} \sum_{\substack{\mu, \nu=1 \\ 1 \leq \mu<\nu}}^{4}\left\{1-\operatorname{Re} \operatorname{Tr}\left(U_{x, \mu, \nu}^{1 \times 1}\right)\right\}+b_{1} \sum_{\substack{\mu, \nu=1 \\ \mu \neq \nu}}^{4}\left\{1-\operatorname{Re} \operatorname{Tr}\left(U_{x, \mu, \nu}^{1 \times 2}\right)\right\}\right),
$$

with $\beta=6 / g_{0}^{2}$ the bare inverse coupling, $b_{1}=-0.331$ and the normalisation condition $b_{0}=1-8 b_{1}$.

The choice of the gauge action is motivated by the non trivial phase structure of Wilsontype fermions at finite values of the lattice spacing. The phase structure of the theory has been extensively studied analytically, by means of chiral perturbation theory [52-58], and numerically [59-64]. These studies provided evidence for a first order phase transition close 
to the chiral point for coarse lattices. This implies that simulations at non-vanishing lattice spacing cannot be performed with pseudoscalar masses below a minimal critical value.

The strength of the phase transition has been found [61,64] to be highly sensitive to the value of the parameter $b_{1}$ in the gauge action in eq. (2.2). Moreover, in [35] it was observed that its strength grows when increasing the number of flavours in the sea from $N_{\mathrm{f}}=2$ to $N_{\mathrm{f}}=2+1+1$, at otherwise fixed physical situation. Numerical studies with our $N_{\mathrm{f}}=2+1+1$ setup have shown that the Iwasaki gauge action, with $b_{1}=-0.331$, provides a smoother dependence of phase transition sensitive quantities on the bare quark mass than the tree-level-improved Symanzik $[65,66]$ gauge action, with $b_{1}=-1 / 12$, chosen for our $N_{\mathrm{f}}=2$ simulations.

Another way to weaken the strength of the phase transition is to modify the covariant derivative in the fermion action by smearing the gauge fields. While the main results of this work do not use smearing of the gauge fields, we report in section 3.7 on our experience when applying a stout smearing [67] procedure, see also [68].

\subsection{Action for the light doublet}

The lattice action for the mass degenerate light doublet $(u, d)$ in the so called twisted basis reads $[3,4]$

$$
S_{l}=a^{4} \sum_{x}\left\{\bar{\chi}_{l}(x)\left[D[U]+m_{0, l}+i \mu_{l} \gamma_{5} \tau_{3}\right] \chi_{l}(x)\right\},
$$

where $m_{0, l}$ is the untwisted bare quark mass, $\mu_{l}$ is the bare twisted light quark mass, $\tau_{3}$ is the third Pauli matrix acting in flavour space and

$$
D[U]=\frac{1}{2}\left[\gamma_{\mu}\left(\nabla_{\mu}+\nabla_{\mu}^{*}\right)-a \nabla_{\mu}^{*} \nabla_{\mu}\right]
$$

is the massless Wilson-Dirac operator. $\nabla_{\mu}$ and $\nabla_{\mu}^{*}$ are the forward and backward gauge covariant difference operators, respectively. Twisted mass light fermions are said to be at maximal twist if the bare untwisted mass $m_{0, l}$ is tuned to its critical value, $m_{\text {crit }}$, the situation we shall reproduce in our simulations. The quark doublet $\chi_{l}=\left(\chi_{u}, \chi_{d}\right)$ in the twisted basis is related by a chiral rotation to the quark doublet in the physical basis

$$
\psi_{l}^{\text {phys }}=e^{\frac{i}{2} \omega_{l} \gamma_{5} \tau_{3}} \chi_{l}, \quad \bar{\psi}_{l}^{\text {phys }}=\bar{\chi}_{l} e^{\frac{i}{2} \omega_{l} \gamma_{5} \tau_{3}},
$$

where the twisting angle $\omega_{l}$ takes the value $\left|\omega_{l}\right| \rightarrow \frac{\pi}{2}$ as $\left|m_{0, l}-m_{\text {crit }}\right| \rightarrow 0$. We shall use the twisted basis throughout this paper.

\subsection{Action for the heavy doublet}

We introduce a dynamical strange quark by adding a twisted heavy mass-split doublet $\chi_{h}=\left(\chi_{c}, \chi_{s}\right)$, thus also introducing a dynamical charm in our framework. As shown in [34], a real quark determinant can in this case be obtained if the mass splitting is taken to be orthogonal in isospin space to the twist direction. We thus choose the construction [33, 34]

$$
S_{h}=a^{4} \sum_{x}\left\{\bar{\chi}_{h}(x)\left[D[U]+m_{0, h}+i \mu_{\sigma} \gamma_{5} \tau_{1}+\mu_{\delta} \tau_{3}\right] \chi_{h}(x)\right\}
$$


where $m_{0, h}$ is the untwisted bare quark mass for the heavy doublet, $\mu_{\sigma}$ the bare twisted mass - the twist is this time along the $\tau_{1}$ direction — and $\mu_{\delta}$ the mass splitting along the $\tau_{3}$ direction.

The bare mass parameters $\mu_{\sigma}$ and $\mu_{\delta}$ of the non-degenerate heavy doublet are related to the physical renormalised strange and charm quark masses via [33]

$$
\begin{aligned}
& \left(m_{s}\right)_{\mathrm{R}}=Z_{\mathrm{P}}^{-1}\left(\mu_{\sigma}-Z_{\mathrm{P}} / Z_{\mathrm{S}} \mu_{\delta}\right), \\
& \left(m_{c}\right)_{\mathrm{R}}=Z_{\mathrm{P}}^{-1}\left(\mu_{\sigma}+Z_{\mathrm{P}} / Z_{\mathrm{S}} \mu_{\delta}\right),
\end{aligned}
$$

where $Z_{\mathrm{P}}$ and $Z_{\mathrm{S}}$ are the renormalisation constants of the pseudoscalar and scalar quark densities, respectively, computed in the massless standard Wilson theory.

A chiral rotation analogous to the one in the light sector transforms the heavy quark doublet from the twisted to the physical basis

$$
\psi_{h}^{\text {phys }}=e^{\frac{i}{2} \omega_{h} \gamma_{5} \tau_{1}} \chi_{h}, \quad \bar{\psi}_{h}^{\text {phys }}=\bar{\chi}_{h} e^{\frac{i}{2} \omega_{h} \gamma_{5} \tau_{1}},
$$

where the twisting angle $\omega_{h}$ takes the value $\left|\omega_{h}\right| \rightarrow \frac{\pi}{2}$ as $\left|m_{0, h}-m_{\text {crit }}\right| \rightarrow 0$.

\section{4 $\mathcal{O}(a)$ improvement at maximal twist}

One of the main advantages of Wilson twisted mass fermions is that by tuning the untwisted bare quark mass to its critical value, automatic $\mathcal{O}(a)$ improvement of physical observables can be achieved.

Tuning the complete $N_{\mathrm{f}}=2+1+1$ action to maximal twist can in principle be performed by independently choosing the bare masses of the light and heavy sectors $a m_{0, l}$ and $a m_{0, h}$, resulting, however, in a quite demanding procedure. On the other hand, properties of the Wilson twisted mass formulation allow for a rather economical, while accurate alternative $[4,34,35]$, where the choice $a m_{0, l}=a m_{0, h} \equiv 1 / 2 \kappa-4$ is made, and the hopping parameter $\kappa$ has been introduced.

Tuning to maximal twist, i.e. $\kappa=\kappa_{\text {crit }}$, is then achieved by choosing a parity odd operator $O$ and determine $a m_{\text {crit }}$ (equivalently $\kappa_{\text {crit }}$ ) such that $O$ has vanishing expectation value. One appropriate quantity is the PCAC light quark mass [29, 60, 61]

$$
m_{\mathrm{PCAC}}=\frac{\sum_{\mathbf{x}}\left\langle\partial_{0} A_{0, l}^{a}(\mathbf{x}, t) P_{l}^{a}(0)\right\rangle}{2 \sum_{\mathbf{x}}\left\langle P_{l}^{a}(\mathbf{x}, t) P_{l}^{a}(0)\right\rangle}, \quad a=1,2,
$$

where

$$
A_{\mu, l}^{a}(x)=\bar{\chi}_{l}(x) \gamma_{\mu} \gamma_{5} \frac{\tau_{a}}{2} \chi_{l}(x), \quad P_{l}^{a}(x)=\bar{\chi}_{l}(x) \gamma_{5} \frac{\tau_{a}}{2} \chi_{l}(x),
$$

and we demand $m_{\mathrm{PCAC}}=0$. For the quenched [25] and the $N_{\mathrm{f}}=2$ case [17], this method has been found to be successful in providing the expected $\mathcal{O}(a)$ improvement and effectively reducing residual $\mathcal{O}\left(a^{2}\right)$ discretisation effects in the region of small quark masses [29].

The numerical precision required for the tuning of $m_{\mathrm{PCAC}}$ to zero has been discussed in [8]. Contrary to the $N_{\mathrm{f}}=2$ case [5, 8], where this tuning was performed once at the minimal value of the twisted light mass considered in the simulations, we now perform the tuning at each value of the twisted light quark mass $\mu_{l}$ and the heavy-doublet quark mass 
parameters $\mu_{\sigma}$ and $\mu_{\delta}$. This obviously leaves more freedom in the choice of light quark masses for future computations.

Although theoretical arguments tell us that $\mathcal{O}(a)$ improvement is at work in our setup, a dedicated continuum scaling study is always required to accurately quantify the actual magnitude of $\mathcal{O}\left(a^{2}\right)$ effects. In section 3.4 we provide a first indication that such effects are indeed small, at least for the here considered light meson sector; currently ongoing computations at a significantly smaller lattice spacing will allow for a continuum limit scaling analysis in this setup.

\section{Simulation details}

\subsection{Simulation ensembles}

We performed simulations at two values of the lattice gauge coupling $\beta=1.90$ and 1.95, corresponding to values of the lattice spacing $a \approx 0.086 \mathrm{fm}$ and $a \approx 0.078 \mathrm{fm}$, respectively. The parameters of each ensemble are reported in table 2 . The charged pion mass $m_{\mathrm{PS}}$ ranges from $270 \mathrm{MeV}$ to $510 \mathrm{MeV}$. Simulated volumes correspond to values of $m_{\mathrm{PS}} L$ ranging from 3.0 to 5.8 , where the smaller volumes served to estimate finite volume effects, see table 3. Physical spatial volumes range from $(1.9 \mathrm{fm})^{3}$ to $(2.8 \mathrm{fm})^{3}$.

As already mentioned, the tuning to $\kappa_{\text {crit }}$ was performed independently for each value of the mass parameters $a \mu_{l}, a \mu_{\sigma}$ and $a \mu_{\delta}$. The mass parameters of the heavy doublet $a \mu_{\sigma}$ and $a \mu_{\delta}$ reported in table 2 are related to the strange and charm quark masses. In particular, they are fixed by requiring the simulated kaon and $D$ meson masses to approximately take their physical values, as discussed in section 3.3. The simulation algorithm used to generate the ensembles includes in the light sector, a Hybrid Monte Carlo algorithm with multiple time scales and mass preconditioning, described in ref. [69], while in the strange-charm sector a polynomial hybrid Monte Carlo (PHMC) algorithm [70-72]; the implementation of ref. [73] is publicly available.

The positivity of the determinant of the Dirac operator is a property of the massdegenerate Wilson twisted mass action, which does not necessarily hold in the non degenerate case for generic values of the mass parameters $\mu_{\sigma}$ and $\mu_{\delta} .{ }^{1}$ The positivity is monitored by measuring the smallest eigenvalue $\lambda_{\mathrm{h} \text {,min }}$ of $Q_{h}^{\dagger} Q_{h}$, where $Q_{h}=\gamma_{5} \tau_{3} D_{h}$ and $D_{h}$ is the Wilson Dirac operator of the non-degenerate twisted mass action in eq. (2.5). We observe that $\lambda_{\mathrm{h}, \mathrm{min}}$ is roughly proportional to the renormalised strange quark mass squared. Since we choose the mass parameters $\mu_{\sigma}$ and $\mu_{\delta}$ such that the strange quark takes its physical value, a spectral gap in the distribution of $Q_{h}^{\dagger} Q_{h}$ is observed, implying that the determinant of $D_{h}$ does not change sign during the simulation. While this is sufficient for the purpose of this study, we shall provide a detailed discussion of this issue in a forthcoming publication.

To generate correlators we use stochastic sources and improve the signal-to-noise ratio by using the "one-end trick", following the techniques also employed in our $N_{\mathrm{f}}=2$ simulations [8]. We have constructed all meson correlators with local (L), fuzzed (F) and Gaussian smeared (S) sources and sinks. The use of smeared or fuzzed sources has stronger

\footnotetext{
${ }^{1}$ Notice however that the positivity of the determinant is guaranteed for $\mu_{\sigma}^{2}>\mu_{\delta}^{2}[33,34]$.
} 


\begin{tabular}{|lcccccc|}
\hline Ensemble & $\beta$ & $\kappa_{\text {crit }}$ & $a \mu_{l}$ & $a \mu_{\sigma}$ & $a \mu_{\delta}$ & $(L / a)^{3} \times T / a$ \\
\hline A30.32 & 1.90 & 0.1632720 & 0.0030 & 0.150 & 0.190 & $32^{3} \times 64$ \\
A40.32 & & 0.1632700 & 0.0040 & & & $32^{3} \times 64$ \\
A40.24 & & 0.1632700 & 0.0040 & & & $24^{3} \times 48$ \\
A40.20 & & 0.1632700 & 0.0040 & & & $20^{3} \times 48$ \\
A50.32 & & 0.1632670 & 0.0050 & & & $32^{3} \times 64$ \\
A60.24 & & 0.1632650 & 0.0060 & & & $24^{3} \times 48$ \\
A80.24 & & 0.1632600 & 0.0080 & & & $24^{3} \times 48$ \\
A100.24 & & 0.1632550 & 0.0100 & & & $24^{3} \times 48$ \\
A100.24s & 0.1631960 & 0.0100 & & 0.197 & $24^{3} \times 48$ \\
\hline B25.32 & 1.95 & 0.1612420 & 0.0025 & 0.135 & 0.170 & $32^{3} \times 64$ \\
B35.32 & & 0.1612400 & 0.0035 & & & $32^{3} \times 64$ \\
B55.32 & 0.1612360 & 0.0055 & & & $32^{3} \times 64$ \\
B75.32 & 0.1612320 & 0.0075 & & & $32^{3} \times 64$ \\
B85.24 & 0.1612312 & 0.0085 & & & $24^{3} \times 48$ \\
\hline
\end{tabular}

Table 2. Summary of the $N_{\mathrm{f}}=2+1+1$ ensembles generated by ETMC at two values of the lattice coupling $\beta=1.90$ and $\beta=1.95$. From left to right, we quote the ensemble name, the value of inverse coupling $\beta$, the estimate of the critical value $\kappa_{\text {crit }}$, the light twisted mass $a \mu_{l}$, the heavy doublet mass parameters $a \mu_{\sigma}$ and $a \mu_{\delta}$ and the volume in units of the lattice spacing. Our notation for the ensemble names corresponds to X. $\mu_{l} . L$, with $\mathrm{X}$ referring to the value of $\beta$ used. The run A100.24s is used to control the tuning of the strange and charm quark masses.

\begin{tabular}{|lccccc|}
\hline Ensemble & $m_{\mathrm{PCAC}} / \mu_{l}$ & $m_{\mathrm{PS}} L$ & $\tau_{\text {int }}(\langle P\rangle)$ & $\tau_{\text {int }}\left(a m_{\mathrm{PS}}\right)$ & $\tau_{\text {int }}\left(a m_{\mathrm{PCAC}}\right)$ \\
\hline A30.32 & $-0.123(87)$ & 3.97 & $118(55)$ & $2.7(4)$ & $46(19)$ \\
A40.32 & $-0.055(55)$ & 4.53 & $103(48)$ & $4.1(7)$ & $51(21)$ \\
A40.24 & $-0.148(83)$ & 3.48 & $132(57)$ & $\leq 2$ & $35(12)$ \\
A40.20 & $-0.051(91)$ & 2.97 & $55(25)$ & $2.9(7)$ & $26(12)$ \\
A50.32 & $0.064(24)$ & 5.05 & $50(19)$ & $3.0(5)$ & $21(7)$ \\
A60.24 & $-0.037(50)$ & 4.15 & $28(8)$ & $2.0(2)$ & $13(4)$ \\
A80.24 & $0.020(19)$ & 4.77 & $23(7)$ & $2.4(3)$ & $10(2)$ \\
A100.24 & $0.025(18)$ & 5.35 & $18(5)$ & $2.3(3)$ & $13(3)$ \\
A100.24s & $0.045(18)$ & 5.31 & $18(5)$ & $6.2(1.1)$ & $18(5)$ \\
\hline B25.32 & $-0.185(69)$ & 3.42 & $65(25)$ & $3.6(6)$ & $26(9)$ \\
B35.32 & $0.009(34)$ & 4.03 & $54(19)$ & $5.5(8)$ & $41(14)$ \\
B55.32 & $-0.069(13)$ & 4.97 & $12(3)$ & $\leq 2$ & $8(2)$ \\
B75.32 & $-0.047(12)$ & 5.77 & $14(4)$ & $3.3(5)$ & $13(3)$ \\
B85.24 & $-0.001(16)$ & 4.66 & $15(4)$ & $2.2(2)$ & $11(2)$ \\
\hline
\end{tabular}

Table 3. For each ensemble, from left to right the values of $m_{\mathrm{PCAC}} / \mu_{l}, m_{\mathrm{PS}} L$, the integrated autocorrelation time of the plaquette, $m_{\mathrm{PS}}$ and $m_{\mathrm{PCAC}}$ in units of the trajectory length. Every ensemble contains 5000 thermalised trajectories of length $\tau=1$, except A40.24 which contains 8000 trajectories. 


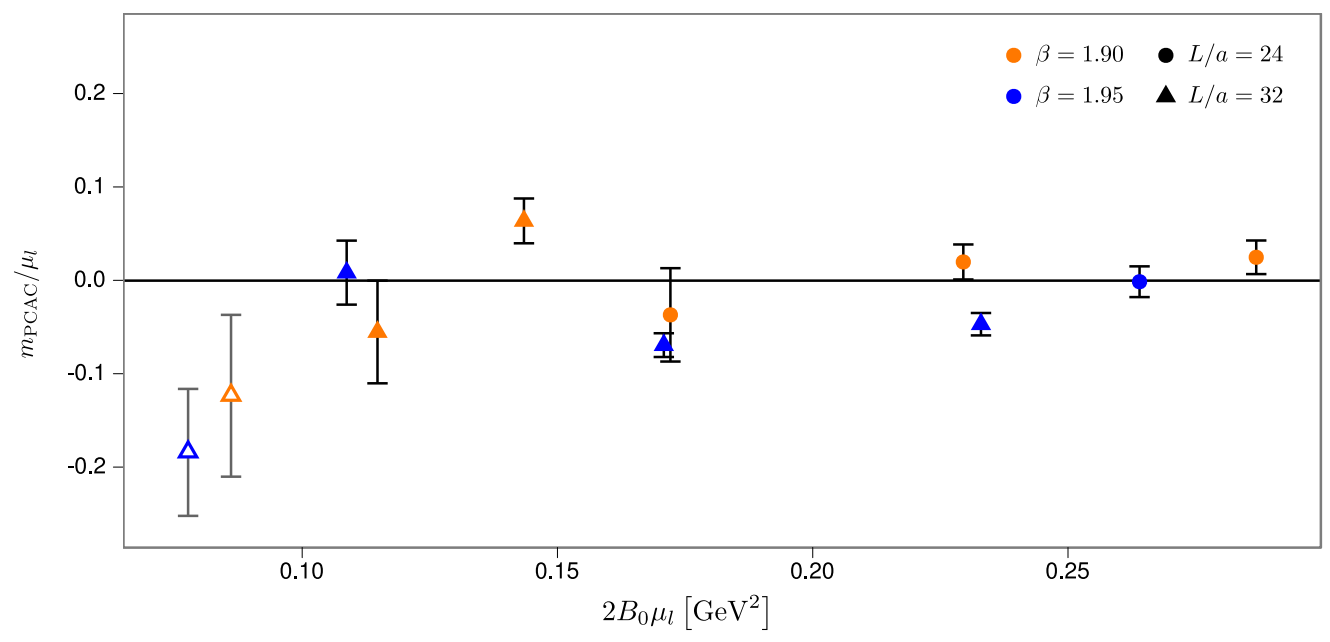

Figure 2. The ratio $m_{\mathrm{PCAC}} / \mu_{l}$ for the ensembles at $\beta=1.90$ and 1.95 at the largest simulated volumes and as a function of $2 B_{0} \mu_{l}$. For both ensembles the ratio $m_{\mathrm{PCAC}} / \mu_{l}$ satisfies the $10 \%$ level criterion, except for the lightest point at $\beta=1.90$ and $\beta=1.95$ (open symbols), also affected by larger statistical errors. We assume $Z_{A}=1$, while the actual value $Z_{A} \lesssim 1$ can only improve all tuning conditions.

impact on the extraction of the kaon and $D$ meson masses; results for the latter are reported in section 3.3, while a companion paper [74] discusses the adopted strategy for the less straightforward determination of these masses in the unitary $N_{\mathrm{f}}=2+1+1$ Wilson twisted mass formalism.

\subsection{Tuning to maximal twist}

To guarantee $\mathcal{O}(a)$ improvement of all physical observables while also avoiding residual $\mathcal{O}\left(a^{2}\right)$ effects with decreasing pion mass, the numerical precision of the tuning to maximal twist - quantified by the deviation from zero of $m_{\mathrm{PCAC}}$ - has to satisfy $\left|Z_{\mathrm{A}} m_{\mathrm{PCAC}} / \mu_{l}\right|_{\mu_{l}, \mu_{\sigma}, \mu_{\delta}} \lesssim a \Lambda_{\mathrm{QCD}}[5,8,17]$. The left-hand side contains the renormalised ratio of the untwisted mass over the twisted light-quark mass. A similar condition should be fulfilled by the error on this ratio. For the current lattice spacings, $a \Lambda_{\mathrm{QCD}} \approx 0.1$, while the values of the axial current renormalisation factor $Z_{\mathrm{A}}$ have not yet been determined. Nevertheless, since $Z_{\mathrm{A}}$ enters as an $\mathcal{O}(1)$ multiplicative prefactor, and it is expected to be $Z_{\mathrm{A}} \lesssim 1$ for our ensembles, ${ }^{2}$ we adopt the conservative choice $Z_{\mathrm{A}}=1$ in verifying the tuning condition.

Satisfying this constraint clearly requires a good statistical accuracy in the determination of the PCAC mass. The values of $m_{\mathrm{PCAC}} / \mu_{l}$ reported in table 3 and shown in figure 2 are well satisfying the tuning condition to maximal twist, with the exception of the lightest mass point at $\beta=1.90$ and $\beta=1.95$. We notice that the autocorrelation time of $m_{\mathrm{PCAC}}$ reported in table 3 grows with decreasing values of the light quark mass $\mu_{l}$, thus rendering

\footnotetext{
${ }^{2}$ Preliminary determinations of $Z_{\mathrm{A}}$ from ongoing dedicated runs with four degenerate light flavours, indicate that $Z_{\mathrm{A}} \sim 0.7-0.8$ for the ensembles considered in this work.
} 


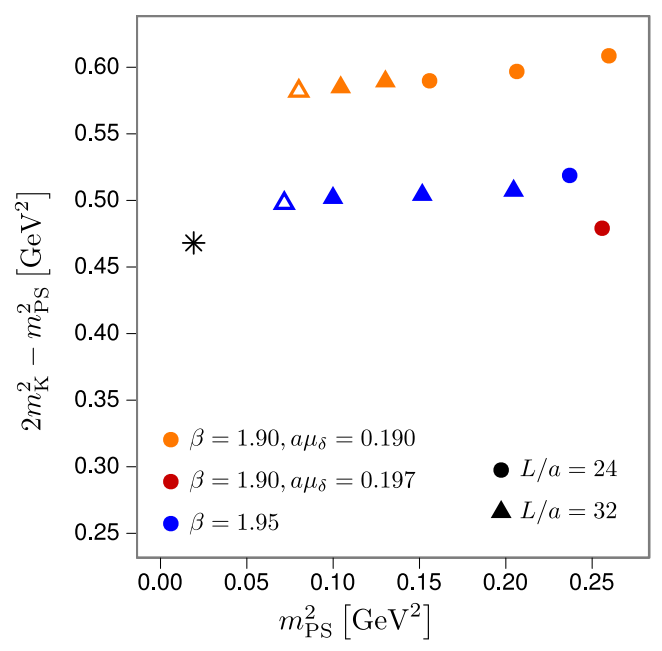

(a)

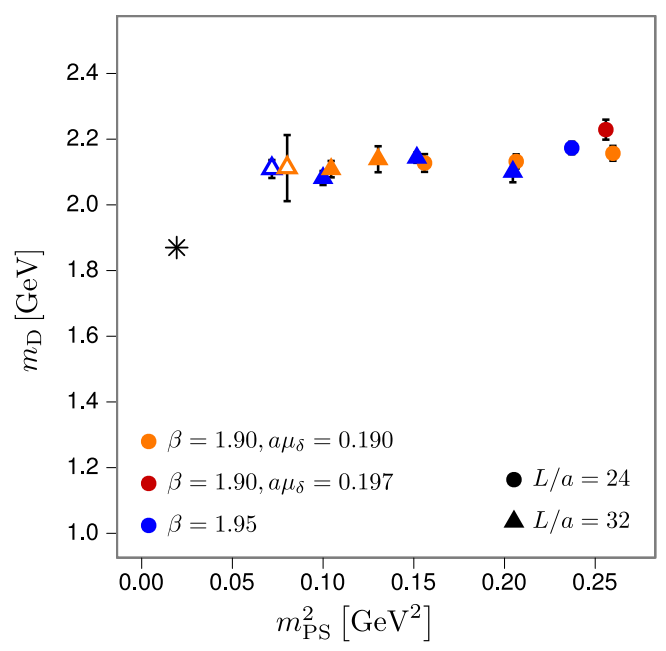

(b)

Figure 3. (a): $2 m_{K}^{2}-m_{\mathrm{PS}}^{2}$, and (b): $m_{D}$, as a function of $m_{\mathrm{PS}}^{2}$, for $\beta=1.95$ (blue) and $\beta=1.90$ (orange). The physical point is shown (black star). The kaon and $D$ meson masses appear to be properly tuned at $\beta=1.95$. The ensembles at $\beta=1.90, \mu_{\delta}=0.190$ have a larger value of the strange quark mass, while the red point at $\beta=1.90, a \mu_{\delta}=0.197$ appears to be well tuned. Data points have been scaled with the lattice spacing $a=0.08585(53) \mathrm{fm}$ for $\beta=1.90$, and $a=0.07820(59) \mathrm{fm}$ for $\beta=1.95$, obtained in this work and where the errors are only statistical.

the tuning more costly for the two lightest points. For the ensemble B25.32, we are currently performing a new simulation aiming at a more accurate tuning to $\kappa_{\text {crit }}$. We are also testing a reweighting procedure [36] in $\kappa$ on the same ensemble, in view of applying it to the other not optimally tuned ensemble A30.32, and to future simulations. In what follows, we use the lightest mass points for consistency checks, and we exclude them from the final $\chi \mathrm{PT}$ fits. We also remind the reader that the small deviations from zero of $a m_{\mathrm{PCAC}}$ will only affect the $\mathcal{O}\left(a^{2}\right)$ lattice discretisation errors of physical observables [8].

\subsection{Tuning of the strange and charm quark masses}

The mass parameters $\mu_{\sigma}$ and $\mu_{\delta}$ in the heavy doublet of the action in eq. (2.5) can in principle be adjusted so as to match the renormalised strange and charm quark masses by use of eq. (2.6). In practise, in this work, we fix the values of $\mu_{\sigma}$ and $\mu_{\delta}$ by requiring that the simulated kaon mass $m_{K}$ and $D$ meson mass $m_{D}$ approximately take their physical values.

A detailed description of the determination of the kaon and $D$ meson masses is separately given in [74], while figures $3 \mathrm{a}$ and $3 \mathrm{~b}$ show the resulting dependence of $\left(2 m_{K}^{2}-m_{\mathrm{PS}}^{2}\right)$ and $m_{D}$ upon the light pseudoscalar mass squared for both ensembles, and compared with the physical point. Table 4 summarises their numerical values, while the corresponding values for $a \mu_{\sigma}$ and $a \mu_{\delta}$ are given in table 2. Observe also that, in order to be able to properly tune the strange and charm quark masses to their physical values, $a \mu_{\sigma}$ must be chosen larger than $a \mu_{\delta}$, since (see eq. (6)) the ratio $Z_{P} / Z_{S}$ is significantly smaller than one [74]. 


\begin{tabular}{|lccc|}
\hline Ensemble & $\beta$ & $a m_{K}$ & $a m_{D}$ \\
\hline A30.32 & 1.90 & $0.25150(29)$ & $0.9230(440)$ \\
A40.32 & & $0.25666(23)$ & $0.9216(109)$ \\
A40.24 & & $0.25884(43)$ & $0.9375(128)$ \\
A40.20 & & $0.26130(135)$ & $0.8701(152)$ \\
A50.32 & & $0.26225(38)$ & $0.9348(173)$ \\
A60.24 & & $0.26695(52)$ & $0.9298(118)$ \\
A80.24 & & $0.27706(61)$ & $0.9319(94)$ \\
A100.24 & & $0.28807(34)$ & $0.9427(99)$ \\
A100.24s & & $0.26502(90)$ & $0.9742(133)$ \\
\hline B25.32 & 1.95 & $0.21240(50)$ & $0.8395(109)$ \\
B35.32 & & $0.21840(28)$ & $0.8286(85)$ \\
B55.32 & $0.22799(34)$ & $0.8532(62)$ \\
B75.32 & & $0.23753(32)$ & $0.8361(127)$ \\
B85.24 & & $0.24476(44)$ & $0.8650(76)$ \\
\hline
\end{tabular}

Table 4. For each ensemble, the values of the kaon mass and the $D$ meson mass as determined in [74].

While the kaon and $D$ meson masses at $\beta=1.95$ are sufficiently well tuned to their physical values, the ensembles at $\beta=1.90$ with $a \mu_{\delta}=0.190$ carry a heavier kaon mass. The latter is instead visibly closer to its physical value for $a \mu_{\delta}=0.197$, as can be inferred from figure 3a. We are currently performing simulations with $a \mu_{\delta}=0.197$ for other light quark masses. Moreover, another set of values of $\mu_{\sigma}$ and $\mu_{\delta}$ are currently being used at $\beta=1.90$ to generate ensembles with a slightly lower $D$ meson mass and a third value of the kaon mass, in order to properly interpolate the lattice data to the physical strange quark mass.

\subsection{Discretisation effects in light-quark observables}

In this section we explore discretisation effects in the analysed light-quark observables. To this aim we also make use of the determination of the chirally extrapolated $r_{0}$ value for our data samples, as discussed in the following section 3.5.

In figures $4 \mathrm{a}$ and $4 \mathrm{~b}$ we study the sensitivity of the charged pion mass and decay constant to possible discretisation effects, by comparing the $N_{\mathrm{f}}=2+1+1$ data at $\beta=1.90$ and $\beta=1.95$ and the results obtained in twisted mass simulations with two dynamical flavours [17]. The alignment of all data points at different values of $\beta$ is in itself an indication of small discretisation effects. The comparison and good agreement with the $N_{\mathrm{f}}=2$ data seems also to suggest no significant dependence upon the inclusion of dynamical strange and charm quarks for these light observables, at least at the present level of accuracy and provided that no cancellations occur due to lattice discretisation effects. However, only a more complete study at significantly different lattice spacings will allow to draw conclusions.

In the same spirit, we show in figure 5 an analogous ratio plot where the nucleon mass data points are included. The alignment of all data and the good extrapolation to the 


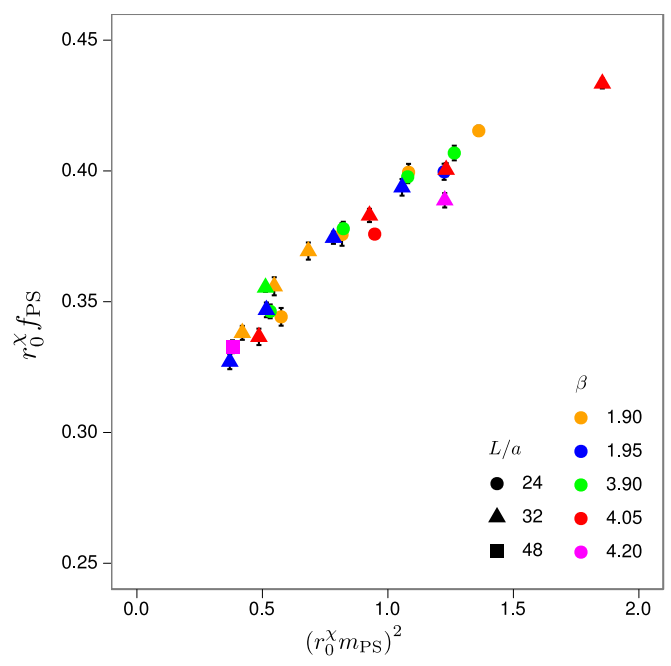

(a)

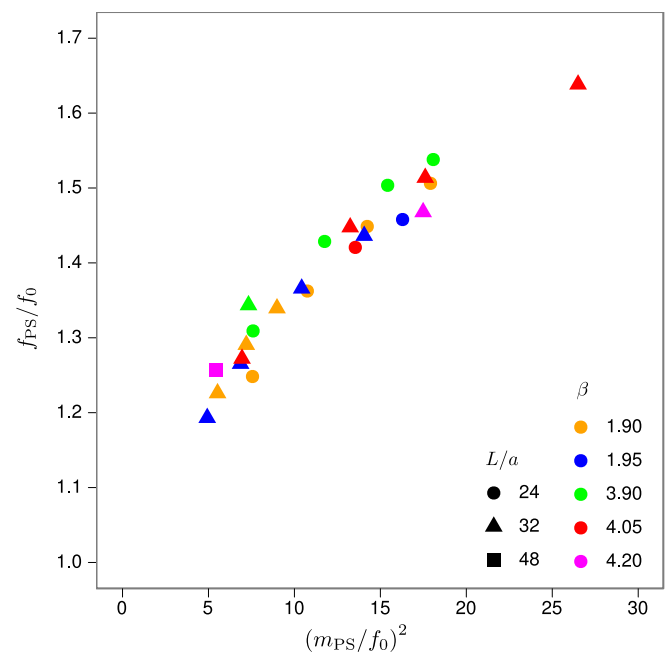

(b)

Figure 4. The quantity $\alpha f_{\mathrm{PS}}$ as a function of $\left(\alpha m_{\mathrm{PS}}\right)^{2}$, with (a) $\alpha=r_{0}^{\chi}$ and (b) $\alpha=1 / f_{0}$, for the $N_{\mathrm{f}}=2+1+1$ data at $\beta=1.90$ and $\beta=1.95$, and for the $N_{\mathrm{f}}=2$ data at $\beta=3.90, \beta=4.05$ and $\beta=4.20$ in [17]. The values of $r_{0}^{\chi}$ for $N_{f}=2+1+1$ are given in tables 1 and 9 .

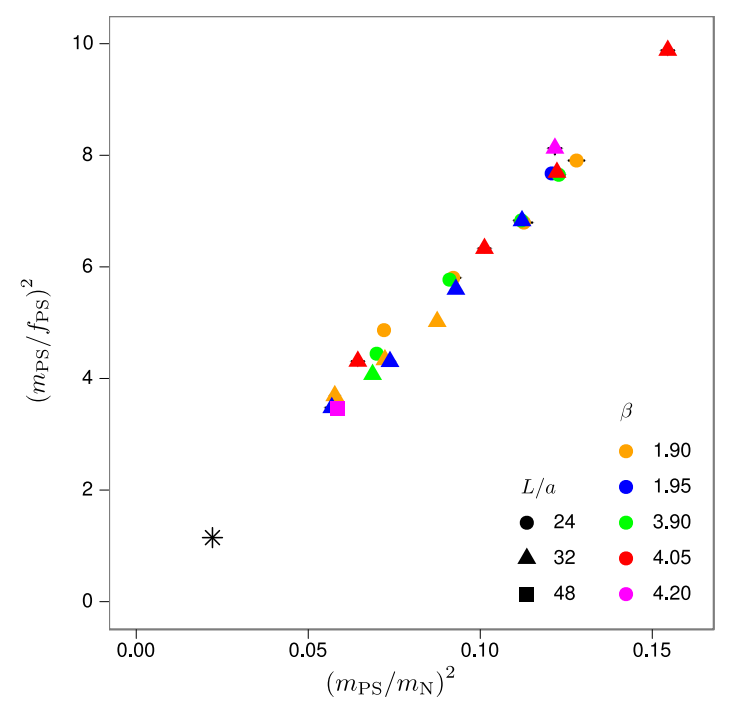

Figure 5. The ratio $m_{\mathrm{PS}}^{2} / f_{\mathrm{PS}}^{2}$ as a function of $m_{\mathrm{PS}}^{2} / m_{N}^{2}$, for the $N_{\mathrm{f}}=2+1+1$ ensembles at $\beta=1.90$ and $\beta=1.95$, compared to the $N_{\mathrm{f}}=2$ data at $\beta=3.90, \beta=4.05$ and $\beta=4.20$ [17]. The physical point is shown (black star). 


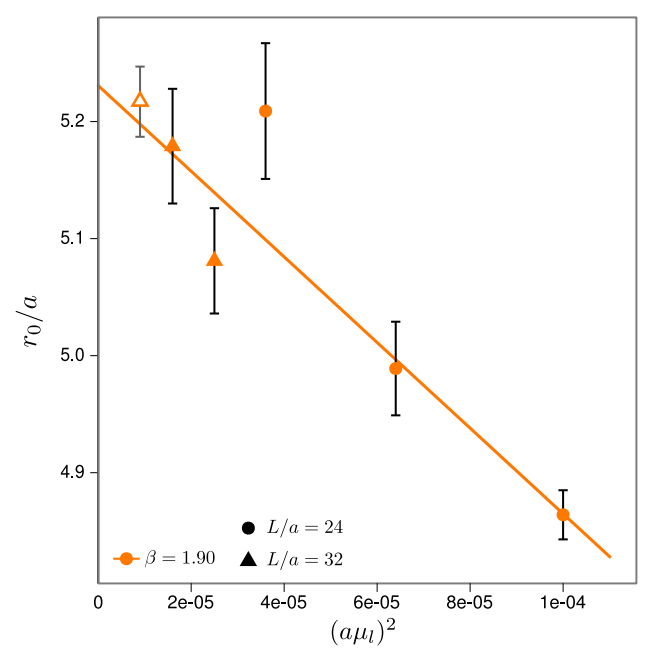

(a)

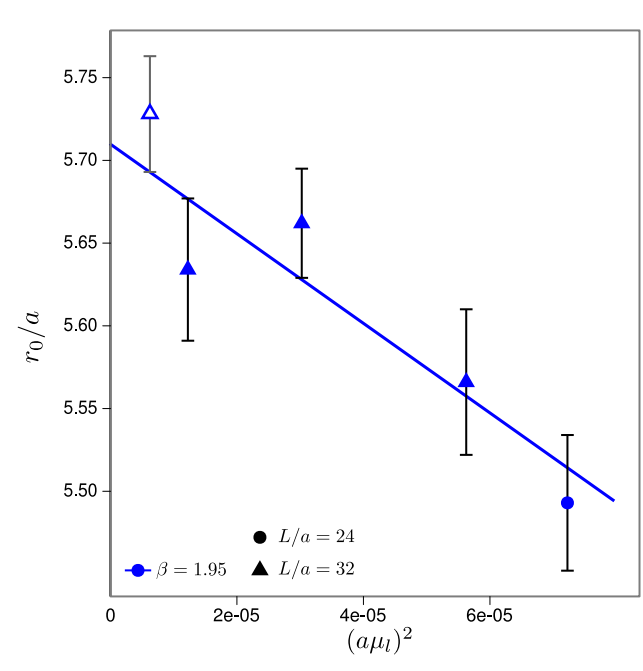

(b)

Figure 6. The Sommer scale $r_{0} / a$ as a function of $\left(a \mu_{l}\right)^{2}$ for $(\mathrm{a}) \beta=1.90$ and $(\mathrm{b}) \beta=1.95$. The lines represent a linear extrapolation in $\left(a \mu_{l}\right)^{2}$ to the chiral limit. The lightest point (open symbol) is not included in the fits and we have always used the largest available volume for a given value of the mass.

physical point is again evident. We defer to future publications the analysis of the baryon spectrum and the study of discretisation effects in strange- and charm-quark observables.

\subsection{The Sommer scale $r_{0}$}

The Sommer scale $r_{0}[75]$ is a purely gluonic quantity extracted from the static inter-quark potential. Since the knowledge of its physical value remains rather imprecise, we use the chirally extrapolated lattice data for $r_{0} / a$ only as an effective way to compare results from different values of the lattice spacing. In this work, the lattice scale is extracted by performing $\chi \mathrm{PT}$ inspired fits to the very precise data for $a f_{\mathrm{PS}}$ and $a m_{\mathrm{PS}}$, and by using the physical values of $m_{\pi}$ and $f_{\pi}$ as inputs.

Figures $6 \mathrm{a}$ and $6 \mathrm{~b}$ display the data for $r_{0} / a$ at both values of the lattice coupling $\beta=1.90$ and 1.95, and as a function of the bare lattice mass squared. The data are reasonably well described by a quadratic dependence, as also previously found for our $N_{\mathrm{f}}=2$ ensembles. For a more detailed discussion of the possible functional forms and their theoretical interpretation see [37]. To extrapolate to the chiral limit, we have performed fits using the largest available volume at each value of the pseudoscalar mass. The chirally extrapolated values for our $N_{\mathrm{f}}=2+1+1$ ensembles are $r_{0}^{\chi} / a=5.231(38)$ at $\beta=1.90$ and $r_{0}^{\chi} / a=5.710(41)$ at $\beta=1.95$, where the lightest points of both ensembles have been excluded from the extrapolation, consistently with the fact that they do not satisfy our most stringent tuning condition to maximal twist.

In order to meaningfully compare the dependence upon the light quark mass at the two different lattice couplings $\beta=1.90$ and 1.95 , we estimated the slope of the functional form 


\begin{tabular}{|lcccc|}
\hline Ensemble & $\beta$ & $r_{0}^{\chi} m_{\mathrm{PS}}^{ \pm}$ & $r_{0}^{\chi} m_{\mathrm{PS}}^{0}$ & $c$ \\
\hline B35.32 & 1.95 & $0.7196(57)$ & $0.388(40)$ & $-12.0(1.1)$ \\
B55.32 & & $0.8861(67)$ & $0.679(40)$ & $-10.6(1.8)$ \\
\hline$B_{6} N_{f}=2$ & 3.90 & $0.7113(66)$ & $0.585(43)$ & $-4.6(1.5)$ \\
$B_{2} N_{f}=2$ & & $0.9001(86)$ & $0.712(54)$ & $-8.6(2.2)$ \\
\hline
\end{tabular}

Table 5. Measurements of the masses of the charged and the neutral pion. We compare runs at $\beta=1.95$ and $N_{f}=2$ runs [17] with comparable lattice spacing and similar charged pion masses in physical units. All masses are reported in units of the chirally extrapolated $r_{0}$ for the same ensemble, see table 9 , and $r_{0}^{\chi} / a=5.316(49)$ for $N_{f}=2$. We also report on the approximate value of $c$, giving the slope of the $a^{2}$ dependence of the pion mass splitting.

$r_{0} / r_{0}^{\chi}=1+c_{r}\left(r_{0}^{\chi} m_{\mathrm{PS}}\right)^{4}$, where the explicit lattice spacing dependence has been removed. We observe a mild dependence on the light quark mass and similar slopes $c_{r}[\beta=1.90]=$ $-0.0379(37)$ and $c_{r}[\beta=1.95]=-0.0234(69)$. It is also worth noticing that the dependence upon the light quark mass of the $N_{\mathrm{f}}=2+1+1$ data and that observed in the $N_{\mathrm{f}}=2$ case [37] are not significantly different.

\subsection{Effects of isospin breaking}

A most delicate aspect of the twisted mass formulation is the breaking of the isospin symmetry. Clear evidence for this breaking has been found in the $N_{\mathrm{f}}=2$ simulations by ETMC when comparing the neutral with the charged pion masses. Indeed, while the discretisation effects in the charged pion were observed to be very small, significant $\mathcal{O}\left(a^{2}\right)$ corrections appear when studying the scaling to the continuum limit of the neutral pion [17]. Notice, however, that similar effects have not been observed in other quantities that are in principle sensitive to isospin breaking but not trivially related to the neutral pion mass. These observations are supported by theoretical considerations detailed in [31,32].

In the $N_{\mathrm{f}}=2+1+1$ case, it turns out that the isospin breaking effect in the mass difference of charged and neutral pion masses is larger than for $N_{\mathrm{f}}=2$ at fixed physical situation, ${ }^{3}$ as can be inferred from table 5 . On the other hand, the same theoretical considerations as in [32] do apply to the case of $N_{\mathrm{f}}=2+1+1$ flavours, and it is expected that the same class of physical observables as for $N_{f}=2$ will not be significantly affected by isospin breaking corrections. Having said that, a careful measure of this effect for each observable or class of observables is anyway mandatory. The increase of the pion mass splitting with increasing the number of flavours in the sea is in line with the observation [35] of a stronger first order phase transition when moving from $N_{\mathrm{f}}=2$ to $N_{\mathrm{f}}=2+1+1$, as discussed in section 2.1. Indeed, the endpoint of the phase transition $[52,53]$ corresponds to the critical value of the light twisted mass $\mu_{l, \mathrm{c}}$ where the neutral pion mass vanishes. The mass difference can be described by $r_{0}^{\chi 2}\left(\left(m_{\mathrm{PS}}^{0}\right)^{2}-\left(m_{\mathrm{PS}}^{ \pm}\right)^{2}\right)=c\left(a / r_{0}^{\chi}\right)^{2}$, where the coefficient $c$ is related to $\mu_{l, \mathrm{c}}[52,53]$ and it is therefore a measure of the strength of the first order phase transition. Hence, a larger value of $c$ means that simulations are to be performed at smaller values of

\footnotetext{
${ }^{3}$ Notice however that different gauge actions are used in the $N_{\mathrm{f}}=2$ and $N_{\mathrm{f}}=2+1+1$ cases as described in section 2.1 .
} 


\begin{tabular}{|lccccccc|}
\hline Ensemble & $\beta$ & $\kappa_{\text {crit }}$ & $a \mu_{l}$ & $a \mu_{\sigma}$ & $a \mu_{\delta}$ & $N_{\text {traj. }}$ & $r_{0} / a$ \\
\hline $\mathrm{A}_{\text {st }} 40.24$ & 1.90 & 0.145512 & 0.0040 & 0.170 & 0.185 & 1500 & $5.304(35)$ \\
$\mathrm{A}_{\text {st }} 60.24$ & & 0.145511 & 0.0060 & & & 3100 & $5.300(37)$ \\
$\mathrm{A}_{\text {st }} 80.24$ & & 0.145510 & 0.0080 & & & 2000 & $5.353(43)$ \\
\hline
\end{tabular}

Table 6. Parameters of the runs with stout smearing on $L / a=24, T / a=48$ lattices. The number of thermalised trajectories with length $\tau=1$ is given by $N_{\text {traj. }}$. The label "st" in the ensemble name refers to the use of stout smearing, compared to the non stout-smeared ensemble in table 2.

\begin{tabular}{|lcccc|}
\hline Ensemble & $a m_{\mathrm{PS}}$ & $a m_{K}$ & $a m_{D}$ & $m_{\mathrm{PCAC}} / \mu_{l}$ \\
\hline $\mathrm{A}_{\mathrm{st}} 40.24$ & $0.12600(93)$ & $0.2479(18)$ & $0.802(27)$ & $0.0175(68)$ \\
$\mathrm{A}_{\mathrm{st}} 60.24$ & $0.14888(78)$ & $0.25338(67)$ & $0.825(26)$ & $0.0017(50)$ \\
$\mathrm{A}_{\mathrm{st}} 80.24$ & $0.17156(69)$ & $0.26198(80)$ & $0.811(12)$ & $0.0138(48)$ \\
\hline
\end{tabular}

Table 7. The masses in lattice units for the ensembles with one level of stout smearing.

the lattice spacing to reach, say, the physical point. Table 5 reports on the values of $m_{\mathrm{PS}}^{ \pm}$, $m_{\mathrm{PS}}^{0}$ and $c$ for some examples taken from the $\beta=1.95$ ensemble and the $N_{f}=2$ ensemble with the closest values of the lattice spacing and physical charged pseudoscalar mass. As anticipated, the coefficient $c$ increases in absolute value from $N_{\mathrm{f}}=2$ to $N_{\mathrm{f}}=2+1+1$.

We are currently performing simulations at a significantly different and lower lattice spacing than the present ensembles. They will allow to determine the slope $c$ for $N_{\mathrm{f}}=2+$ $1+1$ more accurately and to better quantify the conditions to approach the physical point.

\subsection{Stout smeared runs}

In addition to our main simulation ensembles, we also performed runs with stout smeared gauge fields in the lattice fermionic action. The stout smearing as introduced in [67] was designed to have a smearing procedure which is analytic in the unsmeared link variables and hence well suited for HMC-type updating algorithms. In an earlier work with $N_{\mathrm{f}}=2$ quark flavours [68] we showed that using smeared gauge fields in the fermion operator is reducing the strength of the phase transition in twisted quark mass simulations and therefore allows to reach smaller quark masses at a given lattice spacing.

The definition of the stout smeared links can be found in [67], and for the parameter $\rho$ connecting thin to fat gauge links we choose $\rho=0.15$. In principle, such smearing can be iterated several times, with the price of rendering the fermion action delocalised over a larger lattice region. We made a conservative choice to maintain the action well localised and performed a single smearing step. As shown in [68], this kind of smearing does not substantially change the lattice spacing, and for the sake of comparison we thus kept the same value of $\beta$ as in one of the non stout-smeared runs. On the other hand, the hopping parameter has to be tuned again, since the additive renormalisation of the quark mass is expected to be smaller. The parameters of our runs are given in table 6. These runs have been done with the two-step polynomial Hybrid Monte Carlo (TS-PHMC) update algorithm [76]. Results for the hadron masses are collected in table 7, where the quoted 
errors include an estimate of the systematic error induced by variations of the fitting range. The method of estimating and combining statistical and systematic errors for the case of the kaon and $D$ meson masses is described in [74].

As the values of $m_{\mathrm{PCAC}} / \mu_{l}$ in table 7 show, the hopping parameters are well tuned to maximal twist. The masses in the run with smallest light twisted mass $a \mu_{l}=0.0040$ (ensemble $\mathrm{A}_{\mathrm{st}} 40.24$ ) satisfy $r_{0} m_{\mathrm{PS}}=0.668(10), r_{0} m_{K}=1.315(13)$ and $r_{0} m_{D}=4.25(29)$. This means that the pion is lighter than in the corresponding run without stout smearing (see table 8 ) and the kaon and $D$ meson masses are closer to their physical value. The smaller pion mass should be interpreted as due to a quark mass renormalisation factor closer to one. For the same reason the tuned twisted masses in the heavy doublet $a \mu_{\sigma}=0.170$, $a \mu_{\delta}=0.185$ are smaller than in the runs without stout smearing. It is also interesting to compare the mass splitting of the charged and neutral pion between runs with and without stout smearing. For the ensemble $\mathrm{A}_{\mathrm{st}} 60.24$ we obtain a neutral pion mass $r_{0}^{\chi} m_{\mathrm{PS}}^{0}=$ $0.409(34)$ and a charged pion mass $r_{0}^{\chi} m_{\mathrm{PS}}^{ \pm}=0.7861(56)$, in units of the chirally extrapolated value $r_{0}^{\chi} / a=5.280(25)$, providing an estimate of the slope $c=-12.6(0.8)$. Notice that the mass dependence of $r_{0} / a$ in table 6 is reduced as compared to the runs with no stout smearing, and a quadratic dependence on the bare quark mass has been used for the extrapolation to the chiral limit, consistently with the analysis of section 3.5. For the corresponding ensemble A60.24 without stout smearing, using data in tables 8 and 9, we obtain instead $r_{0}^{\chi} m_{\mathrm{PS}}^{0}=0.560(37), r_{0}^{\chi} m_{\mathrm{PS}}^{ \pm}=0.9036(71)$, and a slope $c=-13.8(1.2)$, slightly but not significantly different from the stout-smeared case.

The runs with stout-smeared gauge links show somewhat better characteristics than the ones without stout smearing, but the improvements are not dramatic, at least with one level of stout smearing. More iterations would further accelerate the approach to lighter masses and are expected to further reduce the charged to neutral pion splitting. However, it is a delicate matter to establish how physical observables other than the spectrum will be affected. Based on these considerations and given the present pool of data, the final results in this study are obtained with non stout-smeared simulations.

\section{Results: $f_{\mathrm{PS}}, m_{\mathrm{PS}}$ and chiral fits}

We concentrate in this section on the analysis of the simplest and phenomenologically relevant observables involving up and down valence quarks. These are the light charged pseudoscalar decay constant $f_{\mathrm{PS}}$ and the light charged pseudoscalar mass $m_{\mathrm{PS}}$.

The present simulations with dynamical strange and charm quarks, sitting at, or varying around, their nature given masses, should allow for a good measure of the impact of strange and charm dynamics on the low energy sector of QCD and the electroweak matrix elements. As a first step, one can determine the low energy constants of chiral perturbation theory $(\chi \mathrm{PT})$. The values of $a f_{\mathrm{PS}}$ and $a m_{\mathrm{PS}}$ for our ensembles at $\beta=1.95$ and $\beta=1.90$ are summarised in table 8. In contrast to standard Wilson fermions, an exact lattice Ward identity for maximally twisted mass fermions allows for extracting the charged pseudoscalar 


\begin{tabular}{|lccccc|}
\hline Ensemble & $a \mu_{l}$ & $a m_{\mathrm{PS}}$ & $a f_{\mathrm{PS}}$ & $r_{0} / a$ & $L / a$ \\
\hline A30.32 & 0.0030 & $0.12395(36)(14)$ & $0.06451(35)(3)$ & $5.217(30)$ & 32 \\
A40.32 & 0.0040 & $0.14142(27)(42)$ & $0.06791(18)(4)$ & $5.179(49)$ & 32 \\
A40.24 & 0.0040 & $0.14492(52)(34)$ & $0.06568(34)(7)$ & $5.178(44)$ & 24 \\
A40.20 & 0.0040 & $0.14871(92)(116)$ & $0.06194(65)(23)$ & - & 20 \\
A50.32 & 0.0050 & $0.15796(32)(28)$ & $0.07048(16)(4)$ & $5.081(45)$ & 32 \\
A60.24 & 0.0060 & $0.17275(45)(23)$ & $0.07169(22)(2)$ & $5.209(58)$ & 24 \\
A80.24 & 0.0080 & $0.19875(41)(35)$ & $0.07623(21)(4)$ & $4.989(40)$ & 24 \\
A100.24 & 0.0100 & $0.22293(35)(38)$ & $0.07926(20)(4)$ & $4.864(21)$ & 24 \\
A100.24s & 0.0100 & $0.22125(58)(119)$ & $0.07843(26)(21)$ & $4.918(50)$ & 24 \\
\hline B25.32 & 0.0025 & $0.10680(39)(27)$ & $0.05727(36)(8)$ & $5.728(35)$ & 32 \\
B35.32 & 0.0035 & $0.12602(30)(30)$ & $0.06074(18)(8)$ & $5.634(43)$ & 32 \\
B55.32 & 0.0055 & $0.15518(21)(33)$ & $0.06557(15)(5)$ & $5.662(33)$ & 32 \\
B75.32 & 0.0075 & $0.18020(27)(3)$ & $0.06895(17)(1)$ & $5.566(44)$ & 32 \\
B85.24 & 0.0085 & $0.19396(38)(54)$ & $0.06999(20)(5)$ & $5.493(41)$ & 24 \\
\hline
\end{tabular}

Table 8. Lattice measurements of the charged pseudoscalar mass $a m_{\mathrm{PS}}$, the charged pseudoscalar decay constant $a f_{\mathrm{PS}}$ and the Sommer scale in lattice units $r_{0} / a$ for our two ensembles at $\beta=1.90$ (A set) and $\beta=1.95$ (B set). The value of the light twisted mass $a \mu_{l}$ and the spatial length $L / a$ are also shown. Quoted errors are given as (statistical)(systematic), with the estimate of the systematic error coming from the uncertainty related to the fitting range.

decay constant $f_{\mathrm{PS}}$ from the relation

$$
f_{\mathrm{PS}}=\frac{2 \mu_{l}}{m_{\mathrm{PS}}^{2}}\left|\left\langle 0\left|P_{l}^{1}(0)\right| \pi\right\rangle\right|,
$$

without need to specify any renormalisation factor, since $Z_{\mathrm{P}}=1 / Z_{\mu}[3]$. We have performed fits to $\mathrm{NLO} \mathrm{SU}(2)$ continuum $\chi \mathrm{PT}$ at $\beta=1.95$ and $\beta=1.90$, separately and combined. Results are summarised in table 9.

We thus simultaneously fit our data for the pseudoscalar mass and decay constant to the following formulae, where the contributions $F, D$ and $T$ parametrising finite size corrections, discretisation effects and NNLO $\chi \mathrm{PT}$ effects, respectively, will be discussed below:

$$
\begin{aligned}
m_{\mathrm{PS}}^{2}(L) & =\chi_{\mu}\left(1+\xi l_{3}+D_{m_{\mathrm{PS}}^{2}} a^{2}+\xi^{2} T_{m_{\mathrm{PS}}^{2}}\right) F_{m_{\mathrm{PS}}^{2}} \\
f_{\mathrm{PS}}(L) & =f_{0}\left(1-2 \xi l_{4}+D_{f_{\mathrm{PS}}} a^{2}+\xi^{2} T_{f_{\mathrm{PS}}}\right) F_{f_{\mathrm{PS}}},
\end{aligned}
$$

with the pseudoscalar mass squared at tree level defined as $\chi_{\mu} \equiv 2 B_{0} \mu_{l}$ and the chiral expansion parameter by $\xi \equiv \chi_{\mu} /\left(4 \pi f_{0}\right)^{2}$. The low energy constants $l_{3}$ and $l_{4}$ receive renormalization corrections according to $\bar{l}_{i}=l_{i}+\ln \left[\Lambda^{2} / \chi_{\mu}\right]$, with $\Lambda$ the reference scale. During the fitting procedure, where all quantities are defined in lattice units, we set the reference scale to a single lattice spacing to let its constant logarithmic contribution vanish. Once the scale of the simulation has been set, the low energy constants are rescaled to the scale of the physical pion mass to recover the physical values $\bar{l}_{3}$ and $\bar{l}_{4}$. 
Systematic errors can arise from several sources: finite volume effects, neglecting of higher orders in $\chi \mathrm{PT}$ and finite lattice spacing effects. These different corrections are accounted for explicitly in eq. (4.2). Finite volume corrections are described by the rescaling factors denoted by $F_{m_{\mathrm{PS}}^{2}}$ and $F_{f_{\mathrm{PS}}}$, computed in the continuum theory. Notice that the discretisation effects present in the neutral pion mass, see section 3.6, generate peculiar finite volume corrections which have been recently analysed in ref. [77]. We shall comment on them later. We investigated the effectiveness of one loop continuum $\chi \mathrm{PT}$ finite volume corrections, as first computed in [78], which do not introduce any additional low energy constants. However, the resummed expressions derived by Colangelo, Dürr and Haefeli $(\mathrm{CDH})$ in [79] describe the finite volume effects in our simulations better, be it at the expense of the introduction of two new free parameters, and are thus adopted for this analysis. To $\mathcal{O}\left(\xi^{2}\right)$, these corrections read

$$
\begin{aligned}
F_{m_{\mathrm{PS}}^{2}} & =\left[1-\sum_{n=1}^{\infty} \frac{\rho_{n}}{2 \lambda_{n}}\left(\xi I_{m}^{(2)}+\xi^{2} I_{m}^{(4)}\right)\right]^{2} \\
F_{f_{\mathrm{PS}}} & =1+\sum_{n=1}^{\infty} \frac{\rho_{n}}{\lambda_{n}}\left(\xi I_{f}^{(2)}+\xi^{2} I_{f}^{(4)}\right)
\end{aligned}
$$

with geometric contributions defined as

$$
\begin{aligned}
I_{m}^{(2)}= & -2 K_{1}\left(\lambda_{n}\right) \\
I_{m}^{(4)}= & \left(\frac{101}{9}-\frac{13}{3} \pi+8 l_{1}+\frac{16}{3} l_{2}-5 l_{3}-4 l_{4}\right) K_{1}\left(\lambda_{n}\right)+ \\
& +\left(-\frac{238}{9}+\frac{61}{6} \pi-\frac{16}{3} l_{1}-\frac{64}{3} l_{2}\right) \frac{K_{2}\left(\lambda_{n}\right)}{\lambda_{n}} \\
I_{f}^{(2)}= & -4 K_{1}\left(\lambda_{n}\right) \\
I_{f}^{(4)}= & \left(\frac{29}{18}-\frac{29}{12} \pi+4 l_{1}+\frac{8}{3} l_{2}-6 l_{4}\right) K_{1}\left(\lambda_{n}\right)+ \\
& +\left(-\frac{307}{9}+\frac{391}{24} \pi-\frac{16}{3} l_{1}-\frac{64}{3} l_{2}\right) \frac{K_{2}\left(\lambda_{n}\right)}{\lambda_{n}} .
\end{aligned}
$$

The $K_{i}$ are the modified Bessel functions and the low energy constants $l_{1}$ and $l_{2}$ again receive renormalisation corrections. Equations (4.3) and (4.4) use the shorthand notation $\lambda_{n}=\sqrt{n} m_{\mathrm{PS}} L$. The $\rho_{n}$ in eq. (4.3) are a set of multiplicities, counting the number of ways $n^{2}$ can be distributed over three spatial directions. ${ }^{4}$ Because the finite volume corrections in the case of the volumes used in the chiral fits are fairly small to begin with and subsequent terms quickly decrease, the sums over $n$ can be truncated rather aggressively without real loss of precision. It is therefore unnecessary, in practise, to go beyond the lowest contributions. The parameters $l_{1}$ and $l_{2}$, which are in fact low energy constants appearing at NLO in $\chi \mathrm{PT}$, cannot be determined well from the small finite volume corrections alone. Priors are therefore introduced as additional contributions to the $\chi^{2}$, weighting the deviation of the parameters from their phenomenological values by the

\footnotetext{
${ }^{4}$ These values are straightforwardly precomputed to any order, but are also given in, e.g. [79].
} 
uncertainties in the latter. The values used as priors are -0.4(6) for $\bar{l}_{1}$ and 4.3(1) for $\bar{l}_{2}$ [79], as reported in table 9 . We used the largest available volumes for each ensemble, in the $\chi \mathrm{PT}$ fits. For those points, the difference between the finite volume and the infinite volume values estimated via $\mathrm{CDH}$ formulae for $f_{\mathrm{PS}}$ and $m_{\mathrm{PS}}^{2}$ are within $1 \%$, except for the runs B85.24 and A60.24 (see table 2 and table 8), where they are about 1.5\% for both quantities.

Because of the automatic $\mathcal{O}(a)$ improvement of the twisted mass action at maximal twist, the leading order discretisation artefacts in the chiral formulae of (4.2) are at least of $\mathcal{O}\left(a^{2}\right)$, and $\mathcal{O}\left(a^{2} \mu\right)$ for $m_{\mathrm{PS}}^{2}$. The mass and decay constant of the charged pion have been studied up to NLO $[52,53,58]$ in the context of twisted mass chiral perturbation theory $(\operatorname{tm} \chi \mathrm{PT})$. The regime of quark masses and lattice spacings at which we have performed the simulations is such that $\mu_{l} \gtrsim a \Lambda_{\mathrm{QCD}}^{2}$. In the associated power counting, at maximal twist, the NLO tm $\chi \mathrm{PT}$ expressions for the charged pion mass and decay constant preserve their continuum form. The inclusion of the terms proportional to $D_{m_{\mathrm{PS}}^{2}, f_{\mathrm{PS}}}$, parametrising the lattice artifacts in eq. (4.2), represents an effective way of including sub-leading discretisation effects appearing at NNLO. The finite lattice spacing artefacts can of course not be determined using only data from a single lattice spacing. In addition, including these terms when analysing data with an insufficient range in $a$, may lead to mixing of these degrees of freedom with continuum parameters and thereby destabilise the fits. Hence, these terms were neglected for the separate fits, but included to arrive at a qualitative estimate of these systematic effects in a combined fit to the data at both lattice spacings.

Finite size effects on our data at finite lattice spacing can be analysed in the context of twisted mass chiral perturbation theory as recently proposed in ref. [77]. ${ }^{5}$ However, our present limited set of data with only a small number of different volumes all of them at a single value of the lattice spacing, is not sufficient to apply such an analysis. We plan, however, to perform dedicated runs on different volumes to confront our data to the finite size effect formulae of ref. [77] and to estimate in particular the size of the pion mass splitting in this alternative way.

Finally, results from continuum $\chi \mathrm{PT}$ at NNLO can be included to examine the effect of the truncation at NLO. They are given by

$$
\begin{aligned}
T_{m_{\mathrm{PS}}^{2}} & =\frac{17}{102}\left(49+28 l_{1}+32 l_{2}-9 l_{3}\right)+4 k_{m} \\
T_{f_{\mathrm{PS}}} & =-\frac{1}{6}\left(23+14 l_{1}+16 l_{2}+6 l_{3}-6 l_{4}\right)+4 k_{f} .
\end{aligned}
$$

Two new parameters $k_{m}$ and $k_{f}$ enter these corrections. Again, a limited range of input pion masses may lead to poorly constrained values of these newly introduced parameters, some degree of mixing among different orders and fit instabilities. To retain predictive power and stability, additional priors are given for $k_{m}$ and $k_{f}$, both priors set to $0(1)$, analogously to what is done for $l_{1}$ and $l_{2}$ in the $\mathrm{CDH}$ finite volume corrections.

To set the scale at each lattice spacing, we determine $a \mu_{\text {phys }}$, the value of $a \mu_{l}$ at which the ratio $\sqrt{m_{\mathrm{PS}}^{2}(L=\infty)} / f_{\mathrm{PS}}(L=\infty)$ assumes its physical value. We can then use the value

\footnotetext{
${ }^{5}$ Notice that, in principle, after performing the continuum limit at fixed physical volume, finite size effects can be analysed by means of continuum $\chi \mathrm{PT}$.
} 


\begin{tabular}{|lrrrr|}
\hline & $\beta=1.90$ & $\beta=1.95$ & combined & priors \\
\hline $\bar{l}_{3}$ & $3.435(61)$ & $3.698(73)$ & $3.537(47)$ & - \\
$\bar{l}_{4}$ & $4.773(21)$ & $4.673(25)$ & $4.735(17)$ & - \\
$\bar{l}_{1}$ & $-0.296(104)$ & $-0.430(93)$ & $-0.309(139)$ & $-0.4(6)$ \\
$\bar{l}_{2}$ & $4.260(12)$ & $4.329(15)$ & $4.325(10)$ & $4.3(1)$ \\
$f_{0}[\mathrm{MeV}]$ & $120.956(70)$ & $121.144(83)$ & $121.031(54)$ & - \\
$f_{\pi} / f_{0}$ & $1.0781(18)$ & $1.0764(18)$ & $1.0774(17)$ & - \\
$2 B_{0} \mu_{u, d} / m_{\pi}^{2}$ & $1.029(16)$ & $1.032(21)$ & $1.030(13)$ & - \\
$\left\langle r^{2}\right\rangle_{s}^{\mathrm{NLO}}\left[\mathrm{fm}{ }^{2}\right]$ & $0.7462(43)$ & $0.7237(51)$ & $0.7375(34)$ & - \\
\hline$r_{0}^{\chi} / a(\beta=1.90)$ & $5.231(38)$ & - & $5.231(37)$ & - \\
$r_{0}^{\chi} / a(\beta=1.95)$ & - & $5.710(41)$ & $5.710(42)$ & - \\
$r_{0}^{\chi}(\beta=1.90)[\mathrm{fm}]$ & $0.4491(43)$ & - & $0.4505(40)$ & - \\
$r_{0}^{\chi}(\beta=1.95)[\mathrm{fm}]$ & - & $0.4465(48)$ & $0.4439(39)$ & - \\
$a(\beta=1.90)[\mathrm{fm}]$ & $0.08585(53)$ & - & $0.08612(42)$ & - \\
$a(\beta=1.95)[\mathrm{fm}]$ & - & $0.07820(59)$ & $0.07775(39)$ & - \\
\hline
\end{tabular}

Table 9. Results of the fits to $\mathrm{SU}(2) \chi \mathrm{PT}$ for the ensembles at $\beta=1.95$ and $\beta=1.90$, separate and combined. The largest available volumes are used for each ensemble. Predicted quantities are: the low energy constants $\bar{l}_{3,4}$ (while $\bar{l}_{1,2}$ are introduced with priors), the charged pseudoscalar decay constant in the chiral limit $f_{0}$, the mass ratio $2 B_{0} \mu_{l} / m_{\mathrm{PS}}^{2}$ at the physical point and the pion scalar radius $\left\langle r^{2}\right\rangle_{s}^{\mathrm{NLO}}$. The scale is set by fixing the ratio $f_{\mathrm{PS}}^{[L=\infty]} / m_{\mathrm{PS}}^{[L=\infty]}=f_{\pi} / m_{\pi}=130.4(2) / 135.0$ to its physical value [49]. The chirally extrapolated Sommer scale $r_{0}^{\chi}$ is determined separately and not included in the chiral fits. For a comparison with the $N_{\mathrm{f}}=2$ ETMC results, see [17].

of $f_{\mathrm{PS}}$, or equivalently $m_{\mathrm{PS}}$, to calculate the lattice spacing $a$ in fm from the corresponding physical value. We also perform a chiral fit combining the two different lattice spacings. With only two different values of $\beta$, that are in fact fairly close to each other, a proper continuum limit analysis cannot be performed. Instead, we treat this combined fit as a check on the presence of lattice artefacts and the overall consistency of the data. Without a scaling variable, such as the Sommer scale $r_{0}$, the data from different lattice spacings cannot be directly combined. Rather, the ratios of lattice spacings and light quark mass renormalisation constants $\left(Z_{\mu}=1 / Z_{P}\right)$, as well as the renormalised $B_{0}$ parameter are left free in the fit.

In order to estimate the statistical errors affecting our fitted parameters, we generate at each of the $\mu_{l}$ values 1000 bootstrap samples for $m_{\mathrm{PS}}$ and $f_{\mathrm{PS}}$ extracted from the bare correlators, organised by blocks. For each sample, and combining all masses, we fit $m_{\mathrm{PS}}^{2}$ and $f_{\mathrm{PS}}$ simultaneously as a function of $\mu_{l}$. The parameter set from each of these fits is then a separate bootstrap sample for the purposes of determining the error on our fit results. By resampling $f_{\mathrm{PS}}$ and $m_{\mathrm{PS}}$ on a per-configuration basis, correlations between these quantities are taken into account.

Our final results for the separate and combined fits are summarised in table 9. The $\chi \mathrm{PT}$ fit ansätze provide a satisfactory description of the lattice data, with a $\chi^{2} /$ d.o.f $=$ $5.68 / 4 \simeq 1.4$ at $\beta=1.95, \chi^{2} /$ d.o.f $=4.31 / 6 \simeq 0.7$ at $\beta=1.90$, and $16.9 / 12 \simeq 1.4$ for the 


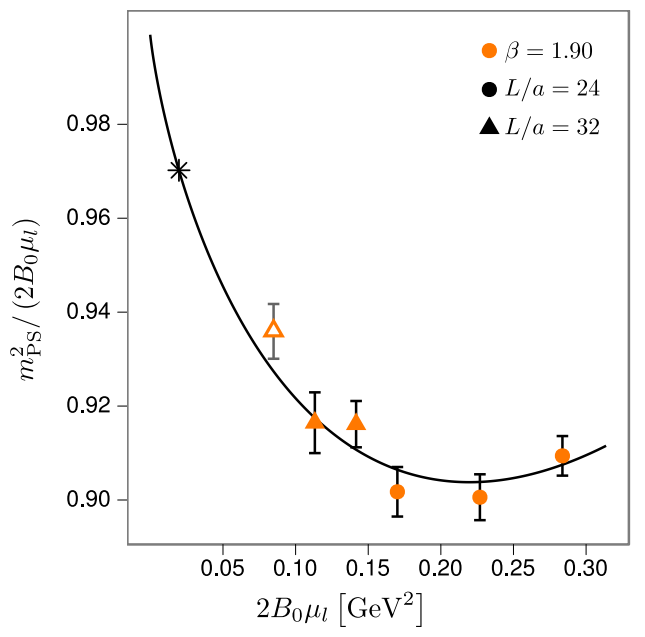

(a)

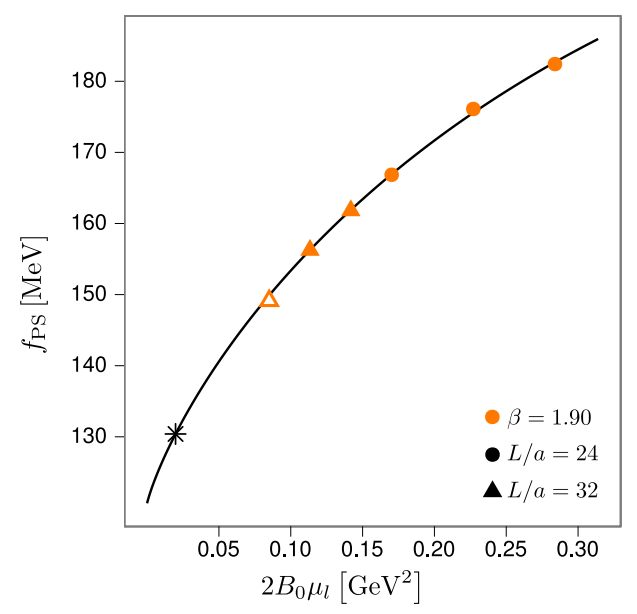

(b)

Figure 7. (a) The charged pseudoscalar mass ratio $m_{\mathrm{PS}}^{2} / 2 B_{0} \mu_{l}$ and (b) the pseudoscalar decay constant $f_{\mathrm{PS}}$ as a function of $2 B_{0} \mu_{l}$, for the ensemble at $\beta=1.90$, fitted to $\mathrm{SU}(2)$ chiral perturbation theory, eq. (4.2). The scale is set by $a \mu_{\mathrm{phys}}$, the value of $a \mu_{l}$ at which the ratio $f_{\mathrm{PS}}^{[L=\infty]} / m_{\mathrm{PS}}^{[L=\infty]}$ assumes its physical value [49] $f_{\pi} / m_{\pi}=130.4(2) / 135.0$ (black star). The light twisted masses used in the fit range from $a \mu_{l}=0.004$ to 0.010 . The lightest point (open symbol) lies outside our most conservative tuning criterion to maximal twist, and is not included in the fit.

combined fit. We also predict the scalar radius of the pion at next to leading order

$$
\left\langle r^{2}\right\rangle_{s}^{\mathrm{NLO}}=\frac{12}{\left(4 \pi f_{0}\right)^{2}}\left(\bar{l}_{4}-\frac{13}{12}\right)
$$

The numerical values in table 9 for the combined fit show a very good agreement with the results from the separate fits, and with errors at the percent level throughout. The fits for $f_{\mathrm{PS}}$ and $m_{\mathrm{PS}}$ at $\beta=1.95$ are displayed in figures $1(\mathrm{a})$ and (b), while in figures 7 (a) and (b) we show the analogous fits at $\beta=1.90$. Figures $8(\mathrm{a})$ and (b) show the results for the fit combining the two $\beta$ values.

The data presented here do not allow yet for a complete account of the systematic effects, but we extract estimates of their magnitude by extending the fits with additional terms as written down in eq. (4.2). Checks were done for $\chi \mathrm{PT}$ NNLO terms and $\mathcal{O}\left(a^{2}\right)$ corrections separately. Including NNLO corrections does not lower the total $\chi^{2}$ of the fit, while we do observe a shift of several standard deviations for the lower order parameters already present in the NLO fit. Using these shifted values to obtain the implied NLO approximation produces fits with much larger values of $\chi^{2}$. We conclude that the current data lack the precision and range in quark masses to constrain NNLO effects, the added degrees of freedom mix with NLO effects and destabilise the fit instead. In practise, we conclude that the systematic error from the truncation of $\chi \mathrm{PT}$ is unobservable at the current level of precision. Inclusion of $\mathcal{O}\left(a^{2}\right)$ corrections leads to similar observations, as the difference between the lattice spacings and the statistical accuracy of the data is too 


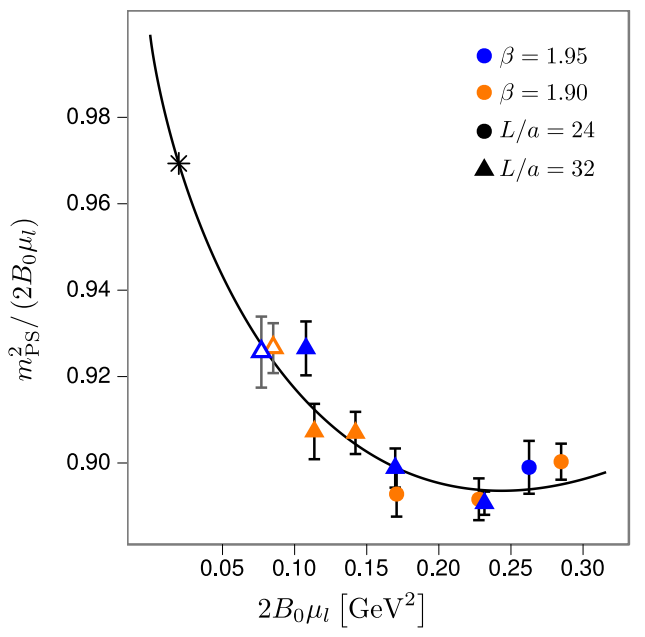

(a)

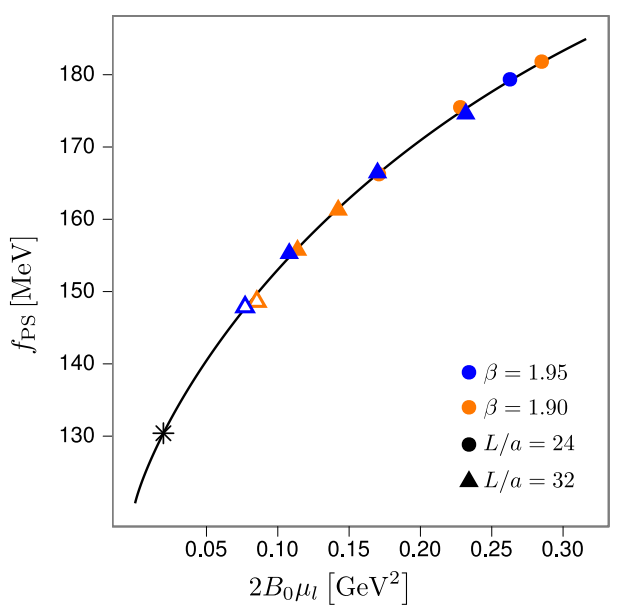

(b)

Figure 8. (a) The charged pseudoscalar mass ratio $\left(m_{\mathrm{PS}} / 2 B_{0} \mu_{l}\right)^{2}$ and (b) the pseudoscalar decay constant $f_{\mathrm{PS}}$ as a function of $2 B_{0} \mu_{l}$, for the combined ensembles at $\beta=1.90$ and $\beta=1.95$, and fitted to eq. (4.2). The scale is set as in figure 7 (black star). The light twisted masses used in the fit range from $a \mu_{l}=0.0035$ to 0.010 . The lightest point at $\beta=1.90$ (open orange symbol) and at $\beta=1.95$ (open blue symbol) lie outside our most conservative tuning criterion to maximal twist, and are not included in the fit.

small to result in a stable fit. The fit mixes $D_{f_{\mathrm{PS}}}$ and $D_{m_{\mathrm{PS}}^{2}}$ on the one hand and $f_{0}, B_{0}$ and the rescaling in the lattice spacing and the quark mass on the other.

The chirally extrapolated Sommer scale $r_{0}^{\chi}$ has been determined separately, using a fit of $r_{0} / a$ with quadratic dependence on the bare light quark mass, as shown in figures $6 \mathrm{a}$ and $6 \mathrm{~b}$, and using the lattice spacing determined by the chiral fits. As also reported in table 9 , the obtained values are $r_{0}^{\chi}=0.4491(43) \mathrm{fm}$ at $\beta=1.90$ and $r_{0}^{\chi}=0.4465(48) \mathrm{fm}$ at $\beta=1.95$, where only statistical errors are quoted. For consistency, we also verified that a combined chiral fit with the inclusion of $r_{0} / a$, as data points and additional fit parameter, gives results anyway in agreement with the strategy adopted here.

For our final estimates of the low energy constants $\bar{l}_{3,4}$ and the chiral value of the pseudoscalar decay constant $f_{0}$ we use the predictions from the $\beta=1.95$ ensemble based on two important observations. First, the strange quark mass in this ensemble is better tuned to the physical value. Secondly a reduced isospin breaking is observed at this finer lattice spacing. The results for the $\beta=1.90$ ensemble and the combined fits serve instead as an estimation of systematic uncertainties. As a result of the current $N_{f}=2+1+1$ simulations we thus quote

$$
\bar{l}_{3}=3.70(7)(26) \quad \bar{l}_{4}=4.67(3)(10),
$$

and $f_{0}=121.14(8)(19) \mathrm{MeV}$, where the first error comes from the chiral fit at $\beta=1.95$, while the second quoted error conservatively accommodates the central values from the $\beta=1.90$ and combined fits as a systematic uncertainty. The predictions for $\bar{l}_{3}$ and $\bar{l}_{4}$ are 
in good agreement and with our two-flavour predictions [17] and with other recent lattice determinations $[2,80]$.

\section{Conclusions and outlook}

In this paper we have presented the first results of lattice QCD simulations with massdegenerate up, down and mass-split strange and charm dynamical quarks using Wilson twisted mass fermions at maximal twist. This study constitutes a first step in our effort to describe low energy strong dynamics and electroweak matrix elements by fully taking into account the effects of a strange and a charm quark.

We have considered ensembles at slightly different lattice spacings simulated with Iwasaki gauge action at $\beta=1.95$ with $a \approx 0.078 \mathrm{fm}$ and $\beta=1.90$ with $a \approx 0.086 \mathrm{fm}$. The charged pseudoscalar masses range from 270 to $510 \mathrm{MeV}$ and we performed fits to $\mathrm{SU}(2)$ chiral perturbation theory with all data at a value of $m_{\mathrm{PS}} L \gtrsim 4$. This analysis provides a prediction for the low energy constants $\bar{l}_{3}=3.70(7)(26)$ and $\bar{l}_{4}=4.67(3)(10)$, for the charged pseudoscalar decay constant in the chiral limit $f_{0}=121.14(8)(19) \mathrm{MeV}$ and for the scalar radius at next-to-leading order $\left\langle r^{2}\right\rangle_{s}^{\mathrm{NLO}}=0.724(5)(23) \mathrm{fm}^{2}$. A companion paper [74] describes the less straightforward determination of the kaon and D-meson masses for the same ensembles.

We have compared our results in the light meson sector with those obtained for $N_{\mathrm{f}}=2$ flavours of maximally twisted mass fermions, ref. [17]. There, an extrapolation to the continuum limit, a study of finite size effects and checks against higher order $\chi \mathrm{PT}$ have been performed, leading to a controlled determination of systematic errors. The comparison we have carried through does not show any significant difference between $N_{\mathrm{f}}=2$ and $N_{\mathrm{f}}=2+1+1$ flavours, at least at the present level of accuracy. These results would suggest that effects of the strange and charm quarks are suppressed for these light observables, as it should be expected. The same comparison has also been used for a first investigation of lattice discretisation errors. As figures $4 \mathrm{a}$ and $4 \mathrm{~b}$ show, the $N_{\mathrm{f}}=2+1+1$ data are completely consistent with the corresponding ones obtained for $N_{\mathrm{f}}=2$, where the discretisation effects have turned out to be very small. Thus, it can be expected that also for the case of $N_{\mathrm{f}}=2+1+1$ flavours the lattice spacing effects will be small, at least for the light meson sector considered here. Notice however that, at the present level of accuracy, there is still the possibility that cancellations occur between physical contributions due to dynamical strange and charm quarks and lattice discretisation effects. A more accurate study at a significantly lower lattice spacing will allow to draw conclusions.

One aspect of the twisted mass formulation is the breaking of isospin symmetry. Its effect is likely to be most pronounced in the lightest sector, where lattice discretisation effects at $\mathcal{O}\left(a^{2}\right)$, affecting the neutral pseudoscalar mass, generate a mass splitting between the charged and the neutral pseudoscalar mesons. While this mass splitting for $N_{f}=$ $2+1+1$ flavours has been found here to be larger than in the $N_{f}=2$ simulations at fixed physical situation, we do not find further effects in other quantities computed so far. This observation is supported by theoretical arguments [31,32] and consistent with our experience in the $N_{f}=2$ flavour case. 
We consider the present results to be encouraging to proceed with the $N_{f}=2+$ $1+1$ flavour research programme of ETMC. In particular, we want to perform the nonperturbative renormalisation with dedicated runs for $N_{f}=4$ mass-degenerate flavours, an activity which we have started already. Furthermore, we want to compute the quark mass dependence of many physical quantities towards the physical point where the pion assumes its experimentally measured value. We are currently performing simulations at a significantly different and lower lattice spacing than the present ensembles. Both strategies, smaller quark masses and smaller lattice spacings, will allow us to estimate systematic effects on a quantitative level and to obtain in this way accurate physical results in our $N_{f}=2+1+1$ flavour simulations with statistical and systematical errors fully under control.

\section{Acknowledgments}

We want to thank the whole ETMC for a very fruitful and enjoyable collaboration. In particular, we gratefully acknowledge valuable suggestions and discussions with Oliver Bär, Benoît Blossier, Roberto Frezzotti, Andreas Nube, Giancarlo Rossi and Enno Scholz.

The computer time for this project was made available to us by the John von Neumann-Institute for Computing (NIC) on the JUMP, Juropa and Jugene systems in Jülich and apeNEXT system in Zeuthen, BG/P and BG/L in Groningen, by BSC on Mare-Nostrum in Barcelona (www.bsc.es), and by the computer resources made available by CNRS on the BlueGene system at GENCI-IDRIS Grant 2009-052271 and CCIN2P3 in Lyon. We thank these computer centres and their staff for all technical advice and help.

V.G. and D.P. thank the MICINN (Spain) for partial support under grant FPA200803373. This work has been supported in part by the DFG Sonderforschungsbereich/ Transregio SFB/TR9-03 and the EU Integrated Infrastructure Initiative Hadron Physics (I3HP) under contract RII3-CT-2004-506078. We also thank the DEISA Consortium (co-funded by the EU, FP6 project 508830), for support within the DEISA Extreme Computing Initiative.

Open Access. This article is distributed under the terms of the Creative Commons Attribution Noncommercial License which permits any noncommercial use, distribution, and reproduction in any medium, provided the original author(s) and source are credited.

\section{References}

[1] K. Jansen, Lattice QCD: a critical status report, PoS(LATTICE 2008) 010 [arXiv:0810.5634] [SPIRES].

[2] E.E. Scholz, Light hadron masses and decay constants, arXiv:0911.2191 [SPIRES].

[3] Alpha collaboration, R. Frezzotti, P.A. Grassi, S. Sint and P. Weisz, Lattice QCD with a chirally twisted mass term, JHEP 08 (2001) 058 [hep-lat/0101001] [SPIRES].

[4] R. Frezzotti and G.C. Rossi, Chirally improving Wilson fermions. I: $\mathcal{O}(a)$ improvement, JHEP 08 (2004) 007 [hep-lat/0306014] [SPIRES].

[5] ETM collaboration, P. Boucaud et al., Dynamical twisted mass fermions with light quarks, Phys. Lett. B 650 (2007) 304 [hep-lat/0701012] [SPIRES]. 
[6] ETM collaboration, B. Blossier et al., Light quark masses and pseudoscalar decay constants from $N_{f}=2$ Lattice QCD with twisted mass fermions, JHEP 04 (2008) 020 [arXiv:0709.4574] [SPIRES].

[7] ETM collaboration, C. Urbach, Lattice QCD with two light Wilson quarks and maximally twisted mass, PoS(LATTICE 2007) 022 [arXiv:0710.1517] [SPIRES].

[8] ETM collaboration, P. Boucaud et al., Dynamical twisted mass fermions with light quarks: simulation and analysis details, Comput. Phys. Commun. 179 (2008) 695 [arXiv:0803.0224] [SPIRES].

[9] K. Cichy, J. Gonzalez Lopez, K. Jansen, A. Kujawa and A. Shindler, Twisted mass, overlap and Creutz fermions: cut-off effects at tree-level of perturbation theory, Nucl. Phys. B 800 (2008) 94 [arXiv:0802.3637] [SPIRES].

[10] ETM collaboration, C. Alexandrou et al., Light baryon masses with dynamical twisted mass fermions, Phys. Rev. D 78 (2008) 014509 [arXiv:0803.3190] [SPIRES].

[11] ETM collaboration, K. Jansen, C. Michael, A. Shindler and M. Wagner, The static-light meson spectrum from twisted mass lattice QCD, JHEP 12 (2008) 058 [arXiv:0810.1843] [SPIRES].

[12] ETM collaboration, K. Jansen, C. Michael and C. Urbach, The $\eta^{\prime}$ meson from lattice QCD, Eur. Phys. J. C 58 (2008) 261 [arXiv:0804.3871] [SPIRES].

[13] R. Frezzotti, V. Lubicz and S. Simula, Electromagnetic form factor of the pion from twisted-mass lattice QCD at $N_{f}=2$, Phys. Rev. D 79 (2009) 074506 [arXiv:0812.4042] [SPIRES].

[14] ETM collaboration, B. Blossier et al., Pseudoscalar decay constants of kaon and D-mesons from $N_{f}=2$ twisted mass lattice QCD, JHEP 07 (2009) 043 [arXiv:0904.0954] [SPIRES].

[15] ETM collaboration, K. Jansen, C. McNeile, C. Michael and C. Urbach, Meson masses and decay constants from unquenched lattice QCD, Phys. Rev. D 80 (2009) 054510 [arXiv:0906.4720] [SPIRES].

[16] ETM collaboration, C. McNeile, C. Michael and C. Urbach, The $\omega-\rho$ meson mass splitting and mixing from lattice QCD, Phys. Lett. B 674 (2009) 286 [arXiv:0902.3897] [SPIRES].

[17] ETM collaboration, R. Baron et al., Light meson physics from maximally twisted mass lattice QCD, arXiv:0911.5061 [SPIRES].

[18] ETM collaboration, C. Alexandrou et al., The low-lying baryon spectrum with two dynamical twisted mass fermions, Phys. Rev. D 80 (2009) 114503 [arXiv:0910.2419] [SPIRES].

[19] ETM collaboration, B. Blossier et al., A proposal for B-physics on current lattices, JHEP 04 (2010) 049 [arXiv:0909.3187] [SPIRES].

[20] ETM collaboration, B. Blossier, M. Wagner and O. Pene, Lattice calculation of the Isgur-Wise functions $\tau_{1 / 2}$ and $\tau_{3 / 2}$ with dynamical quarks, JHEP 06 (2009) 022 [arXiv:0903.2298] [SPIRES].

[21] ETM collaboration, M. Constantinou et al., Non-perturbative renormalization of quark bilinear operators with $N_{f}=2$ (tmQCD) Wilson fermions and the tree-level improved gauge action, arXiv: 1004.1115 [SPIRES].

[22] ETM collaboration, C. Michael, A. Shindler and M. Wagner, The continuum limit of the static-light meson spectrum, arXiv:1004.4235 [SPIRES]. 
[23] A. Shindler, Twisted mass lattice QCD, Phys. Rept. 461 (2008) 37 [arXiv:0707.4093] [SPIRES].

[24] XLF collaboration, K. Jansen, A. Shindler, C. Urbach and I. Wetzorke, Scaling test for Wilson twisted mass QCD, Phys. Lett. B 586 (2004) 432 [hep-lat/0312013] [SPIRES].

[25] XLF collaboration, K. Jansen, M. Papinutto, A. Shindler, C. Urbach and I. Wetzorke, Light quarks with twisted mass fermions, Phys. Lett. B 619 (2005) 184 [hep-lat/0503031] [SPIRES].

[26] XLF collaboration, K. Jansen, M. Papinutto, A. Shindler, C. Urbach and I. Wetzorke, Quenched scaling of Wilson twisted mass fermions, JHEP 09 (2005) 071 [hep-lat/0507010] [SPIRES].

[27] A.M. Abdel-Rehim, R. Lewis and R.M. Woloshyn, Spectrum of quenched twisted mass lattice QCD at maximal twist, Phys. Rev. D 71 (2005) 094505 [hep-lat/0503007] [SPIRES].

[28] ETM collaboration, P. Dimopoulos, R. Frezzotti, G. Herdoiza, C. Urbach and U. Wenger, Scaling and low energy constants in lattice $Q C D$ with $N_{f}=2$ maximally twisted Wilson quarks, PoS(LATTICE 2007) 102 [arXiv:0710.2498] [SPIRES].

[29] R. Frezzotti, G. Martinelli, M. Papinutto and G.C. Rossi, Reducing cutoff effects in maximally twisted lattice QCD close to the chiral limit, JHEP 04 (2006) 038 [hep-lat/0503034] [SPIRES].

[30] XLF collaboration, K. Jansen et al., Flavour breaking effects of Wilson twisted mass fermions, Phys. Lett. B 624 (2005) 334 [hep-lat/0507032] [SPIRES].

[31] R. Frezzotti and G. Rossi, $\mathcal{O}\left(a^{2}\right)$ cutoff effects in Wilson fermion simulations, PoS (LATTICE 2007) 277 [arXiv: 0710.2492] [SPIRES].

[32] ETM collaboration, P. Dimopoulos, R. Frezzotti, C. Michael, G.C. Rossi and C. Urbach, $\mathcal{O}\left(a^{2}\right)$ cutoff effects in lattice Wilson fermion simulations, Phys. Rev. D 81 (2010) 034509 [arXiv: 0908.0451] [SPIRES].

[33] R. Frezzotti and G.C. Rossi, Chirally improving Wilson fermions. II: four-quark operators, JHEP 10 (2004) 070 [hep-lat/0407002] [SPIRES].

[34] R. Frezzotti and G.C. Rossi, Twisted-mass lattice QCD with mass non-degenerate quarks, Nucl. Phys. (Proc. Suppl.) 128 (2004) 193 [hep-lat/0311008] [SPIRES].

[35] T. Chiarappa et al., Numerical simulation of $Q C D$ with $u, d, s$ and $c$ quarks in the twisted-mass Wilson formulation, Eur. Phys. J. C 50 (2007) 373 [hep-lat/0606011] [SPIRES].

[36] ETM collaboration, R. Baron et al., Status of ETMC simulations with $N_{f}=2+1+1$ twisted mass fermions, PoS(LATTICE 2008) 094 [arXiv: 0810.3807] [SPIRES].

[37] ETM collaboration, R. Baron et al., First results of ETMC simulations with $N_{f}=2+1+1$ maximally twisted mass fermions, PoS (LAT2009) 104 [arXiv:0911.5244] [SPIRES].

[38] MILC collaboration, A. Bazavov et al., HISQ action in dynamical simulations, PoS(LATTICE 2008) 033 [arXiv: 0903.0874] [SPIRES].

[39] MILC collaboration, A. Bazavov et al., Progress on four flavor QCD with the HISQ action, PoS (LAT2009) 123 [arXiv: 0911.0869] [SPIRES].

[40] MILC collaboration, A. Bazavov et al., Scaling studies of QCD with the dynamical HISQ action, arXiv: 1004.0342 [SPIRES]. 
[41] L. Del Debbio, L. Giusti, M. Lüscher, R. Petronzio and N. Tantalo, QCD with light Wilson quarks on fine lattices (I): first experiences and physics results, JHEP 02 (2007) 056 [hep-lat/0610059] [SPIRES].

[42] RBC-UKQCD collaboration, C. Allton et al., Physical results from $2+1$ flavor domain wall QCD and $\mathrm{SU}(2)$ chiral perturbation theory, Phys. Rev. D 78 (2008) 114509 [arXiv: 0804.0473] [SPIRES].

[43] JLQCD collaboration, S. Aoki et al., Two-flavor QCD simulation with exact chiral symmetry, Phys. Rev. D 78 (2008) 014508 [arXiv:0803.3197] [SPIRES].

[44] JLQCD and TWQCD collaborations, J. Noaki et al., Convergence of the chiral expansion in two-flavor lattice QCD, Phys. Rev. Lett. 101 (2008) 202004 [arXiv:0806. 0894] [SPIRES].

[45] S. Dürr et al., Ab-initio determination of light hadron masses, Science 322 (2008) 1224 [arXiv:0906.3599] [SPIRES].

[46] PACS-CS collaboration, S. Aoki et al., Physical point simulation in $2+1$ flavor lattice QCD, Phys. Rev. D 81 (2010) 074503 [arXiv:0911.2561] [SPIRES].

[47] PACS-CS collaboration, K.I. Ishikawa et al., SU(2) and SU(3) chiral perturbation theory analyses on baryon masses in $2+1$ flavor lattice QCD, Phys. Rev. D 80 (2009) 054502 [arXiv: 0905.0962] [SPIRES].

[48] A. Bazavov et al., Full nonperturbative QCD simulations with $2+1$ flavors of improved staggered quarks, arXiv:0903.3598 [SPIRES].

[49] Particle Data Group collaboration, C. Amsler et al., Review of particle physics, Phys. Lett. B 667 (2008) 1 [SPIRES].

[50] Y. Iwasaki, Renormalization group analysis of lattice theories and improved lattice action: two-dimensional nonlinear $O(N) \sigma$-model, Nucl. Phys. B 258 (1985) 141 [SPIRES].

[51] Y. Iwasaki, K. Kanaya, T. Kaneko and T. Yoshie, Scaling in SU(3) pure gauge theory with a renormalization group improved action, Phys. Rev. D 56 (1997) 151 [hep-lat/9610023] [SPIRES].

[52] G. Münster and C. Schmidt, Chiral perturbation theory for lattice QCD with a twisted mass term, Europhys. Lett. 66 (2004) 652 [hep-lat/0311032] [SPIRES].

[53] S.R. Sharpe and J.M.S. Wu, Twisted mass chiral perturbation theory at next-to-leading order, Phys. Rev. D 71 (2005) 074501 [hep-lat/0411021] [SPIRES].

[54] S. Aoki and O. Bär, Twisted-mass $Q C D, \mathcal{O}(a)$ improvement and Wilson chiral perturbation theory, Phys. Rev. D 70 (2004) 116011 [hep-lat/0409006] [SPIRES].

[55] S.R. Sharpe and J.M.S. Wu, The phase diagram of twisted mass lattice QCD, Phys. Rev. D 70 (2004) 094029 [hep-lat/0407025] [SPIRES].

[56] G. Münster, On the phase structure of twisted mass lattice QCD, JHEP 09 (2004) 035 [hep-lat/0407006] [SPIRES].

[57] G. Münster, C. Schmidt and E.E. Scholz, Chiral perturbation theory for twisted mass QCD, Nucl. Phys. (Proc. Suppl.) 140 (2005) 320 [hep-lat/0409066] [SPIRES].

[58] L. Scorzato, Pion mass splitting and phase structure in twisted mass QCD, Eur. Phys. J. C 37 (2004) 445 [hep-lat/0407023] [SPIRES]. 
[59] F. Farchioni et al., Twisted mass quarks and the phase structure of lattice QCD, Eur. Phys. J. C 39 (2005) 421 [hep-lat/0406039] [SPIRES].

[60] F. Farchioni et al., Exploring the phase structure of lattice QCD with twisted mass quarks, Nucl. Phys. (Proc. Suppl.) 140 (2005) 240 [hep-lat/0409098] [SPIRES].

[61] F. Farchioni et al., The phase structure of lattice QCD with Wilson quarks and renormalization group improved gluons, Eur. Phys. J. C 42 (2005) 73 [hep-lat/0410031] [SPIRES].

[62] F. Farchioni et al., Dynamical twisted mass fermions, PoS (LAT2005) 072 [hep-lat/0509131] [SPIRES].

[63] F. Farchioni et al., Numerical simulations with two flavours of twisted-mass Wilson quarks and DBW2 gauge action, Eur. Phys. J. C 47 (2006) 453 [hep-lat/0512017] [SPIRES].

[64] F. Farchioni et al., Lattice spacing dependence of the first order phase transition for dynamical twisted mass fermions, Phys. Lett. B 624 (2005) 324 [hep-lat/0506025] [SPIRES].

[65] P. Weisz, Continuum limit improved lattice action for pure Yang-Mills theory. 1, Nucl. Phys. B 212 (1983) 1 [SPIRES].

[66] P. Weisz and R. Wohlert, Continuum limit improved lattice action for pure Yang-Mills theory. 2, Nucl. Phys. B 236 (1984) 397 [Erratum ibid. B 247 (1984) 544] [SPIRES].

[67] C. Morningstar and M.J. Peardon, Analytic smearing of SU(3) link variables in lattice QCD, Phys. Rev. D 69 (2004) 054501 [hep-lat/0311018] [SPIRES].

[68] K. Jansen et al., Stout smearing for twisted mass fermions, PoS(LATTICE 2007) 036 [arXiv: 0709.4434] [SPIRES].

[69] C. Urbach, K. Jansen, A. Shindler and U. Wenger, HMC algorithm with multiple time scale integration and mass preconditioning, Comput. Phys. Commun. 174 (2006) 87 [hep-lat/0506011] [SPIRES].

[70] R. Frezzotti and K. Jansen, A polynomial hybrid Monte Carlo algorithm, Phys. Lett. B 402 (1997) 328 [hep-lat/9702016] [SPIRES].

[71] R. Frezzotti and K. Jansen, The PHMC algorithm for simulations of dynamical fermions. I: description and properties, Nucl. Phys. B 555 (1999) 395 [hep-lat/9808011] [SPIRES].

[72] T. Chiarappa, R. Frezzotti and C. Urbach, $A(P) H M C$ algorithm for $N_{f}=2+1+1$ flavours of twisted mass fermions, PoS (LAT2005) 103 [hep-lat/0509154] [SPIRES].

[73] K. Jansen and C. Urbach, tmLQCD: a program suite to simulate Wilson twisted mass lattice QCD, Comput. Phys. Commun. 180 (2009) 2717 [arXiv:0905.3331] [SPIRES].

[74] ETM collaboration, R. Baron et al., Computing $K$ and $D$ meson masses with $N_{f}=2+1+1$ twisted mass lattice QCD, arXiv:1005.2042 [SPIRES].

[75] R. Sommer, A new way to set the energy scale in lattice gauge theories and its applications to the static force and $\alpha_{s}$ in $\mathrm{SU}(2)$ Yang-Mills theory, Nucl. Phys. B 411 (1994) 839 [hep-lat/9310022] [SPIRES].

[76] I. Montvay and E. Scholz, Updating algorithms with multi-step stochastic correction, Phys. Lett. B 623 (2005) 73 [hep-lat/0506006] [SPIRES]. 
[77] G. Colangelo, U. Wenger and J.M.S. Wu, Twisted mass finite volume effects, arXiv: 1003.0847 [SPIRES].

[78] J. Gasser and H. Leutwyler, Light quarks at low temperatures, Phys. Lett. B 184 (1987) 83 [SPIRES].

[79] G. Colangelo, S. Dürr and C. Haefeli, Finite volume effects for meson masses and decay constants, Nucl. Phys. B 721 (2005) 136 [hep-lat/0503014] [SPIRES].

[80] S. Necco, Chiral low-energy constants from lattice QCD, PoS (CONFINEMENT8) 024 [arXiv: 0901.4257] [SPIRES]. 